\title{
BANACH-VALUED MULTILINEAR SINGULAR INTEGRALS WITH MODULATION INVARIANCE
}

\author{
FRANCESCO DI PLINIO, KANGWEI LI, HENRI MARTIKAINEN, AND EMIL VUORINEN
}

\begin{abstract}
Aвstract. We prove that the class of trilinear multiplier forms with singularity over a one dimensional subspace, including the bilinear Hilbert transform, admit bounded $L^{p}$ extension to triples of intermediate UMD spaces. No other assumption, for instance of Rademacher maximal function type, is made on the triple of UMD spaces. Among the novelties in our analysis is an extension of the phase-space projection technique to the UMD-valued setting. This is then employed to obtain appropriate single tree estimates by appealing to the UMD-valued bound for bilinear Calderón-Zygmund operators recently obtained by the same authors.
\end{abstract}

\section{INTRODUCTION AND MAIN RESULTS}

Let $\mathcal{X}_{k}, k=1,2,3$, be Banach spaces with a trilinear contraction $\mathcal{X}_{1} \times \mathcal{X}_{2} \times \mathcal{X}_{3} \rightarrow \mathbb{C}$ which we denote by $\left(e_{1}, e_{2}, e_{3}\right) \mapsto e_{1} e_{2} e_{3}=\prod_{k=1}^{3} e_{k}$. To a multiplier $m$ defined on the orthogonal complement $\Gamma$ of $(1,1,1) \in \mathbb{R}^{3}$, we may associate the trilinear form

$$
\Lambda_{m}\left(f_{1}, f_{2}, f_{3}\right)=\int_{\Gamma} m(\xi)\left(\prod_{k=1}^{3} \widehat{f_{k}}(\xi)\right) \mathrm{d} \xi
$$

acting on functions $f_{k} \in \mathcal{S}(\mathbb{R}) \otimes \mathcal{X}_{k}, k=1,2,3$, where the former is the Schwartz class. This article is concerned with multipliers $m$ whose singularity lies on a one-dimensional

(F. Di Plinio) Department of Mathematics, Washington University in St. Louis, One Brookings Drive, Sт. Louis, MO 63130-4899, USA

(K. Li) Center for Applied Mathematics, Tianjin University, Weijin Road 92, 300072 Tianjin, China

(H. Martikainen) Department of Mathematics and Statistics, University of Helsinki, P.O.B. 68, FI-00014 University of Helsinki, Finland

(E. Vuorinen) Centre for Mathematical Sciences, University of Lund, P.O.B. 118, 22100 Lund, Sweden

E-mail addresses: francesco.diplinio@wustl.edu, kangwei.nku@gmail.com,

henri.martikainen@helsinki.fi, j.e.vuorin@gmail.com.

2010 Mathematics Subject Classification. 42B20.

Key words and phrases. bilinear Hilbert transform, singular integrals, modulation invariance, UMD spaces, phase-space projections, interpolation spaces.

F. Di Plinio has been partially supported by the National Science Foundation under the grant NSF-DMS1800628.

H. Martikainen was supported by the Academy of Finland through the grants 294840, 306901 and 327271 and by the three-year research grant 75160010 of the University of Helsinki. He is a member of the Finnish Centre of Excellence in Analysis and Dynamics Research supported by the Academy of Finland (project No. 307333).

E. Vuorinen was supported by the Jenny and Antti Wihuri Foundation. 
subspace perpendicular to a unit vector $\beta \in \Gamma$ which is nondegenerate in the sense that

$$
\Delta(\beta):=\min _{j \neq k}\left|\beta_{j}-\beta_{k}\right|>0,
$$

and satisfies for all multi-indices $\alpha$

$$
\sup _{\xi \in \Gamma}\left(\operatorname{dist}\left(\xi, \beta^{\perp}\right)\right)^{\alpha}\left|\partial_{\alpha} m(\xi)\right| \lesssim_{\alpha} 1 .
$$

Assumption (1) is a $\beta^{\perp}$-modulation invariant version of the Coifman-Meyer condition. This class includes the bilinear Hilbert transform with parameter $\beta$, whose dual trilinear multiplier form may be obtained by choosing

$$
m(\xi)=\operatorname{sgn}(\xi \cdot \beta)
$$

The bilinear Hilbert transform

$$
T\left(f_{1}, f_{2}\right)(x)=\int f_{1}\left(x-b_{1} t\right) f_{2}\left(x-b_{2} t\right) \frac{\mathrm{d} t}{t}, \quad b_{j}=\beta_{j}-\beta_{3}, j=1,2,
$$

was first introduced by Calderón within the context of $L^{p}$ estimates for the first commutator of the Cauchy integral along Lipschitz curves. The celebrated articles of Lacey and Thiele $[25,26]$ contain the first proof of $L^{p}$ estimates for the bilinear Hilbert transform, while more general multipliers of the class (1) were treated by Muscalu, Tao and Thiele [27].

1.1. Main results. In this article, we prove that the trilinear multiplier forms (1), where $m$ is a multiplier of the class (1), admit $L^{p}$-bounded extensions to triples of intermediate UMD Banach spaces. This terminology has been introduced by Hytönen and Lacey [19,20]: we repeat this definition below and send to [17] and references therein for background and generalities on UMD Banach spaces.

Let $2 \leq q \leq \infty$ and $\mathcal{X}_{0}, \mathcal{X}_{1}$ be a couple of compatible Banach spaces, with $\mathcal{X}_{0}$ being a UMD space and $\mathcal{X}_{1}$ being a Hilbert space. We say that the Banach space $\mathcal{X}$ is $q$-intermediate UMD if

$$
X=\left[X_{0}, X_{1}\right]_{\frac{2}{q}}
$$

namely, $\mathcal{X}$ is the complex interpolation of a UMD Banach space with a Hilbert space. Such Banach space $\mathcal{X}$ is automatically a UMD space. Notice that $\mathcal{X}$ is $q$-intermediate UMD if and only if its Banach dual $X^{\prime}$ is.

The precise statement of our main result is as follows.

1.3. Theorem. Let $\mathcal{X}_{j}, j=1,2,3$, be Banach spaces with Banach duals $\boldsymbol{Y}_{j}=\mathcal{X}_{j}^{\prime}$ and suppose that each $\mathcal{X}_{j}$ is $q_{X_{j}}$-intermediate UMD. Assume that

$$
\rho=\sum_{j=1}^{3} \frac{1}{q_{X_{j}}}-1>0 .
$$

Let $\sigma$ be any permutation of $\{1,2,3\}, m$ be a multiplier satisfying (1) and $T_{m, \sigma}$ denote the adjoint bilinear operator to (1) acting on pairs of $\mathcal{X}_{\sigma(1)}, \mathcal{X}_{\sigma(2)}$ functions. Then

$$
\left\|T_{m, \sigma}\left(f_{1}, f_{2}\right)\right\|_{L^{\frac{p_{1} p_{2}}{p_{1}+p_{2}}\left(\mathbb{R} ; \boldsymbol{y}_{\sigma(3)}\right)}} \lesssim\left\|f_{\sigma(1)}\right\|_{L^{p_{1}\left(\mathbb{R} ; X_{\sigma(1)}\right.}}\left\|f_{\sigma(2)}\right\|_{L^{p_{2}\left(\mathbb{R} ; \mathcal{X}_{\sigma(2)}\right)}}
$$


whenever

$$
1<p_{1}, p_{2} \leq \infty, \quad\left(p_{1}, p_{2}\right) \neq(\infty, \infty), \quad\left(\frac{1}{p_{1}}, \frac{1}{p_{2}}\right) \in \operatorname{int}(\mathcal{H}) .
$$

Here $\mathcal{H}$ is the hexagon with vertices $A, B, C, D, E, F$ as follows:

$$
\begin{array}{ll}
A:\left(\frac{1}{q_{X_{1}}}-\rho q_{X_{3}}, \frac{1}{q_{X_{2}}}\right), & D:\left(\frac{1}{q_{X_{1}}}+\rho q_{X_{1}}-\rho, \frac{1}{q_{X_{2}}}\right), \\
B:\left(\frac{1}{q_{X_{1}}}, \frac{1}{q_{X_{2}}}-\rho q_{X_{3}}\right), & E:\left(\frac{1}{q_{X_{1}}}, \frac{1}{q_{X_{2}}}+\rho q_{X_{2}}-\rho\right), \\
C:\left(\frac{1}{q_{X_{1}}}+\rho q_{X_{1}}-\rho, \frac{1}{q_{X_{2}}}-\rho q_{X_{1}}\right), & F:\left(\frac{1}{q_{X_{1}}}-\rho q_{X_{2}}, \frac{1}{q_{X_{2}}}+\rho q_{X_{2}}-\rho\right) .
\end{array}
$$

We note in passing that if condition (1.1) holds, the range $\operatorname{int}(\mathcal{H})$ is nonempty and in particular contains the region

$$
q_{X_{k}}<p_{k}<\infty, k=1,2,3, \quad p_{3}:=\left(\frac{p_{1} p_{2}}{p_{1}+p_{2}}\right)^{\prime}
$$

which is the analogue of the local $L^{2}$ range for the scalar case, see [25]. In addition, we point out that $\operatorname{int}(\mathcal{H})$ may contain quasi-Banach pairs $\left(p_{1}, p_{2}\right)$, that is, pairs with $\frac{p_{1} p_{2}}{p_{1}+p_{2}}<1$. This is easier to see by particularizing Theorem 1.1 to the case

$$
X_{1}=\mathcal{X}, X_{2}=\mathcal{X}^{\prime}, \mathcal{X}_{3}=\mathbb{C},
$$

as in the following corollary. Herein, quasi-Banach estimates are available if $2<q<3$.

1.6. Corollary. Let $\mathcal{X}$ be a $q$-intermediate UMD space and define the trilinear contraction

$$
(\mathfrak{x}, \phi, \lambda) \in \mathcal{X} \times \mathcal{X}^{\prime} \times \mathbb{C} \mapsto \lambda \phi(\mathfrak{x}) .
$$

Let $m$ be a multiplier satisfying (1) and $T_{m}$ denote the adjoint bilinear operator to (1) acting on pairs of $\mathcal{X}, \mathcal{X}^{\prime}$ functions.

Suppose that $2 \leq q \leq 3$. Then

$$
\left\|T_{m}\left(f_{1}, f_{2}\right)\right\|_{L^{\frac{p_{1} p_{2}}{p_{1}+p_{2}}(\mathbb{R})}} \lesssim\left\|f_{1}\right\|_{L^{p_{1}(\mathbb{R} ; \mathcal{X})}}\left\|f_{\sigma(2)}\right\|_{L^{p_{2}\left(\mathbb{R} ; X^{\prime}\right)}}
$$

whenever

$$
1+\frac{(q-1)(q-2)}{q(5-q)-2}<p_{1}, p_{2} \leq \infty, \quad \frac{2}{3}\left(1+\frac{q-2}{5-q}\right)<\frac{p_{1} p_{2}}{p_{1}+p_{2}}<\frac{q}{q-2} .
$$

If $3<q<4$, then (1.2) holds true if, in addition to (1.2), the condition

is verified.

$$
\frac{q^{2}-3 q+1}{q}<\min _{(u, v) \in\{(1,2),(2,1)\}}\left\{\frac{q-1}{p_{u}}+\frac{q-2}{p_{v}}\right\}
$$

Theorem 1.1 and Corollary 1.2 further the rather recent line of research on the extension of singular operators with modulation invariance properties to UMD Banach spaces without any UMD lattice function space structure, or lattice structure altogether: a prototypical example are noncommutative $L^{p}$ spaces such as the reflexive Schatten-von Neumann subclasses of the algebra of bounded operators on a Hilbert space. This line of research was initiated by Hytönen and Lacey in their proof of boundedness of the Carleson maximal partial Fourier sum operator for intermediate UMD spaces in the Walsh [20] and Fourier 
[19] setting; see also [22] for Walsh-Carleson variation norm bounds. Subsequently, the same authors and Parissis [21] proved the analogue of Theorem 1.1 for the Walsh model of the bilinear Hilbert transform. In fact, the range of exponents $\operatorname{int}(\mathcal{H})$ is the same as the one obtained therein for the Walsh model, see [21, Theorem 9.3]. Results in the vein of [21] were recently reproved by Amenta and Uraltsev in [2] as a byproduct of novel Banach-valued outer $L^{p}$ space embeddings for the Walsh wave packet transform.

The theory of UMD-valued linear singular integrals of Calderón-Zygmund type is rooted in the works by Burkholder [6] and Bourgain [5] among others, and has been extensively developed since then, see for instance $[7,14-16,18,23,24,33]$ and the monograph [17]. Recent advances have concerned the UMD extension of multilinear CaldéronZygmund operators [11-13]. The above mentioned references deal with generic UMD spaces, as opposed to lattices, and thus develop fundamentally different techniques from those of the classical vector-valued theory of e.g. Benedek, Calderón and Panzone, Fefferman-Stein, Rubio de Francia, which are strictly tied to $A_{p}$-type weighted norm inequalities. In a similar contrast, the present article combines novel technical tools in UMD-valued time frequency analysis to the UMD interpolation space idea of [19] in order to deal with multilinear modulation invariant operators on non-lattice UMD spaces, which are out of reach for typical lattice-based techniques.

Nevertheless, a systematic function space-valued theory for (1) is quite recent. The first proof of $\ell^{p}$-valued bounds for the bilinear Hilbert transform in a wide range of exponents is due to P. Silva [30]. In [30], those estimates have been employed to obtain bounds for the biparameter bilinear operator obtained by tensoring the bilinear Hilbert transform with a Coifman-Meyer multiplier. Several extensions and refinements of [30] have since appeared, see e.g. [1, 4, 8,9]. In general, as Corollary 1.2 demonstrates, Theorem 1.1 is outside the scope of the above references, although it does imply a strict subset of the $\ell^{p}$ estimates of [30]. We send to [2,21] for a detailed discussion of this point.

However, to stress the difference with the results of [30] and followups, we would like to showcase here a further application of Theorem 1.1 to a triple of non-function, non-lattice UMD Banach spaces, in addition to that of Corollary 1.2. In the corollary that follows we denote by $S^{p}, 1 \leq p<\infty$ the $p$-th Schatten-von Neumann class, namely, the subspace of the von Neumann algebra $\mathcal{B}(H)$ of linear bounded operators on a separable Hilbert space defined by the norm

$$
\|A\|_{S^{p}}=\left\|s_{n}(A)\right\|_{\ell p(n \in \mathbb{N})}
$$

where $\left\{s_{n}(A): n \in \mathbb{N}\right\}$ is the sequence of singular values of $A$, that is eigenvalues of the Hermitian operator $|A|=\sqrt{A^{*} A}$. Notice that the classes $S^{p}$ are increasingly nested with $p$ and that the trilinear form

$$
\left(A_{1}, A_{2}, A_{3}\right) \in S^{t_{1}} \times S^{t_{2}} \times S^{t_{3}} \mapsto \operatorname{trace}\left(A_{1} A_{2} A_{3}\right)
$$

is a contraction provided that

$$
\sum_{k=1}^{3} \frac{1}{t_{k}} \geq 1 .
$$


1.10. Corollary. Suppose that the exponents $1<t_{1}, t_{2}, t_{3}<\infty$ satisfy, in addition to (1.1), the conditions

$$
\rho=\sum_{k=1}^{3} \frac{1}{\max \left\{t_{k},\left(t_{k}\right)^{\prime}\right\}}-1>0 .
$$

Let $\sigma$ be a permutation of $\{1,2,3\}$, and $m$ be a multiplier satisfying (1) Then the corresponding adjoint bilinear operator $T_{m, \sigma}$ maps

$$
T_{m, \sigma}: L^{p_{1}}\left(\mathbb{R} ; S^{t_{\sigma(1)}}\right) \times L^{p_{2}}\left(\mathbb{R} ; S^{t_{\sigma(2)}}\right) \rightarrow L^{\frac{p_{1} p_{2}}{p_{1}+p_{2}}}\left(\mathbb{R} ; S^{t_{\sigma(3)}^{\prime}}\right)
$$

boundedly for all $p_{1}, p_{2}$ specified by (1.1).

Corollary 1.3 is obtained from Theorem 1.1 by noticing that $S^{p}, 1<p<\infty$ is intermediate UMD of exponent $\max \left\{p, p^{\prime}\right\}$. Similar statements may be obtained for more general non-commutative $L^{p}$ spaces. We send to [29] for comprehensive definitions and background: a quick harmonic analyst-friendly introduction is given in [12, Section 3].

1.2. Techniques of proof and novelties. The standard proofs of $L^{p}$-bounds for the scalarvalued versions of the forms $\Lambda_{m}$ in (1) are articulated in roughly three separate moments. The first is to realize that the forms (1) lie in the convex hull of suitable discretized model versions, the so-called tri-tile forms, displaying the same modulation and translation invariance properties of the condition (1): this step extends verbatim to the vector-valued case. We may thus focus on $L^{p}$-bounds for the model sums.

An essential step of the proof is the decomposition of the model operators into (discretized) multipliers which are adapted to a certain fixed top frequency and localized in space to a top interval. These tree model sums are essentially trilinear Calderón-Zygmund forms. The contribution of each tree is then controlled by localized space-frequency norms of the involved functions, the so-called energies (or sizes). This bound is referred to as tree estimate.

In the vector-valued case, this second step has to be adapted in a nontrivial and novel fashion. First of all, the vector-valued energies, introduced in (3.3), (3.3) must be defined in terms of local $q$-norms of (linear) tree operators rather than simply $\ell^{2}$ sums of wavelet coefficients coming from each tree. We do so by means of a technical modification of the approach in [19]. Second, and most important, we obtain an effective tree estimate by replacing the involved functions with vector-valued phase-space projections to the spacefrequency support of the tree. This extension of the scalar-valued phase-space projections of e.g. $[10,28]$ to UMD spaces, which may be of independent interest, is carried out in Proposition 4.1, and is the main technical novelty of the article. The tree model sum acts on the phase-space projections roughly as a trilinear $\mathrm{CZ}$ multiplier operator, and the $L^{p}$-norms of the constructed projections are controlled by the corresponding energies. These observations may be used in conjunction with the $L^{p}$-bound for UMD extensions of bilinear CZ operators, recently obtained by the authors of this paper in [12], to produce the tree estimate of Lemma 3.3.

Finally, the recomposition of the bounds obtained for each tree into a global estimate relies on almost-orthogonality considerations. To export this almost-orthogonality to the vector-valued scenario, we rely, as in previous literature $[2,19,21]$, on the $q$-intermediate property of the involved spaces $\mathcal{X}$. This step is carried out in Lemma 3.2. As every known example of UMD space is $q$-intermediate for some $q$, this assumption may seem 
harmless. However, unlike the linear setting of [19], it is the combined $q$-intermediate type of the three spaces that introduces the restriction (1.1) and influences the range $\operatorname{int}(\mathcal{H})$ in Theorem 1.1. Further investigation on the necessity and on possible weakening of the $q$-intermediate assumptions are left for future work.

Plan of the paper. Section 2 contains the preliminary material needed to define the model tri-tile forms. Section 3 presents the outline of the proof of Theorem 1.1: in particular, the definitions of trees, vector-valued energies as well as the statement of the energy and tree lemmata, respectively Lemma 3.2 and 3.3. Section 4 contains the proof of the tree Lemma 3.3 via the reduction to the phase-space projection Proposition 4.1. The proof of the latter proposition is developed in Section 5. Section 6 contains the proof of the energy Lemma 3.2, while Lemma 3.1 is proved in the concluding Section 7.

Remark. In the final stages of preparation of the present manuscript, the authors learned of the work [3] by Amenta and Uraltsev. These authors obtain a simultaneous and independent version of Theorem 1.1, focused on the bilinear Hilbert transform in the Banach range of exponents, under the same intermediate space condition (1.1). Interestingly, the methods employed in [3] are rather different from ours: the use of phase-space projections and of the UMD Calderón-Zygmund estimates from [12] is replaced by outer embeddings for the vector-valued wave packet transform involving telescoping (defect) energies.

The authors want to thank Alex Amenta and Gennady Uraltsev for sharing their preprint and for interesting discussions on the subject.

\section{SPACE-FREQUENCY MODEL SUMS}

2.1. Notation. While our estimates are valid in any ambient space $\mathbb{R}^{d}$, we work with $d=1$ to avoid unnecessary notational proliferation. However, we adopt $d$-dimensional terminology and notation whenever possible. For instance, we write $B_{r}(x)=\{y \in \mathbb{R}$ : $|y-x|<r\}$ and simply $B_{r}$ in place of $B_{r}(0)$. Whenever possible, spatial and frequency 1-dimensional cubes are indicated respectively by $I, \omega$. The center and sidelength of a 1-dimensional cube $I$ are respectively denoted by $c(I), \ell(I)$. We use the Japanese bracket notation $\langle x\rangle=\sqrt{1+|x|^{2}}$.

If $m$ is a bounded function on $\mathbb{R}$, we denote both the corresponding $L^{2}(\mathbb{R})$-bounded Fourier multiplier operator and its trivial extension to a Banach space $\mathcal{X}$ as

$$
T_{m} f(x)=\int \widehat{f}(\xi) m(\xi) \mathrm{e}^{i x \xi} \mathrm{d} \xi, \quad x \in \mathbb{R} .
$$

When $\mathcal{X}$ is a Banach space, we keep denoting by $T$ the trivial extension $T \otimes \operatorname{Id}$ of a linear operator $T$.

2.2. Frequency-localized indicators. Indicator functions, e.g. of intervals, possess perfect localization in space but poor frequency decay. We come to defining frequencylocalized approximations of indicator functions by weakening such spatial localization to polynomial decay. Let $J, K$ be large fixed integers, $c_{0}$ be a small positive constant and $\eta: \mathbb{R} \rightarrow[0, \infty)$ have the following properties:

$$
\text { supp } \widehat{\eta} \subset B_{2^{-2 J}}, \quad c\langle x\rangle^{-2 K} \leq \eta(x) \leq\langle x\rangle^{-2 K} \quad \forall x \in \mathbb{R} .
$$


We rescale $\eta$ at frequency scale $2^{j J}, \eta_{j}:=2^{j J} \eta\left(2^{j J} \cdot\right)$, and for $E \subset \mathbb{R}, j \in \mathbb{Z}$ we introduce

$$
\chi_{E, j}=\mathbf{1}_{E} * \eta_{j}
$$

whose frequency support is contained in $B_{2^{(j-2) J}}$. This definition will be of particular interest to us when $I$ is a $J$-dyadic interval, that is, $\ell(I)=2^{-j J}$ for some $j \in \mathbb{Z}$. In that case we simply write $\chi_{I}$ in place of $\chi_{I, j}$. Notice that, for $\chi=\chi_{I}, N=2 K, \delta=2^{-2 J}, c=c_{0}$ there holds

$$
\begin{aligned}
& \operatorname{supp} \hat{\chi} \subset B_{\delta \ell(I)^{-1}} ; \\
& \chi \text { real-valued, } \quad c\left\langle\frac{x-c(I)}{\ell(I)}\right\rangle^{-N} \leq \chi(x) \leq C\left\langle\frac{x-c(I)}{\ell(I)}\right\rangle^{-N}, \quad x \in \mathbb{R} .
\end{aligned}
$$

More generally, we denote by $X_{I}(N, \delta, c, C)$ the class of functions satisfying conditions (2.2)-(2.2). If $\psi$ instead satisfies (2.2) and

$$
|\psi(x)| \leq C\left\langle\frac{x-c(I)}{\ell(I)}\right\rangle^{-N}, \quad x \in \mathbb{R}
$$

in place of the more stringent (2.2), we say that $\psi \in \Psi_{I}(N, \delta, C)$. Obviously, the inclusion $X_{I}(N, \delta, c, C) \subset \Psi_{I}(N, \delta, C)$ holds.

It is important to notice that if $I, I^{\prime}$ are $A$-comparable intervals, that is $I \subset A I^{\prime}, I^{\prime} \subset A I$ and $\chi \in X_{I}(N, \delta, c, C)$, then $\chi \in X_{I^{\prime}}\left(N^{\prime}, \delta^{\prime}, c^{\prime}, C^{\prime}\right)$ as well, for suitable values of $N^{\prime}, \delta^{\prime}, c^{\prime}, C^{\prime}$ depending only on the comparability constant $A$ and on $N, \delta, C, C$. A similar statement applies to the classes $\Psi_{I}(N, \delta, C)$.

Let now $\omega$ be a $J$-dyadic frequency interval and $\beta \geq 1$ : the latter parameter will take moderate values, $1 \leq \beta \ll 2^{J}$. The class $M_{\omega}(\beta)$ will consist of those smooth functions $m$ with supp $m \subset \beta \omega$ and adapted to $\beta \omega$ in the sense that

$$
\sup _{|\alpha| \leq C} \sup _{\xi \in \mathbb{R}} \beta^{-\alpha} \ell(\omega)^{\alpha}\left|\partial_{\xi}^{\alpha} m(\xi)\right| \leq 1 \text {. }
$$

As customary, we will work with tiles $t=I_{t} \times \omega_{t} \in \mathbb{R} \times \mathbb{R}$, namely the cartesian product of dyadic intervals in $\mathbb{R}$ of reciprocal length, to specify time-frequency localizations. In the remainder of the article, we restrict ourselves to considering J-dyadic intervals. Mimicking rank 1 projections in a Hilbert space, we may define classes of multiplier operators adapted to each tile $t$ as follows. Whenever $\psi \in \Psi_{I_{t}}(N, \delta, C), m \in M_{\omega_{t}}(\beta)$, the operator

$$
S_{t} f(x)=\psi(x) T_{m} f(x)=\int_{\mathbb{R}} \psi(x) m(\xi) \widehat{f}(\xi) \mathrm{e}^{i x \cdot \xi} \mathrm{d} \xi
$$

is said to belong to the class $\mathbb{S}_{t}(N, \delta, \beta, c, C)$ of $t$-localized operators. From the rapid decay of the kernel of $T_{m}$ and the adaptedness of $\psi$ it follows that

$$
\left|S_{t} f(x)\right|_{\chi} \lesssim\left\langle\frac{\operatorname{dist}\left(x, I_{t}\right)}{\ell\left(I_{t}\right)}\right\rangle^{-100} M\left(|f|_{X}\right)(x), \quad x \in \mathbb{R} .
$$

Here $\mathcal{X}$ may be any Banach space, not necessarily UMD.

2.3. Remark. In the remainder of the paper, the values $N, \delta, \beta, c, C$ will vary within a fixed range

$$
c \geq \bar{c}, \quad C \leq \bar{C}, \quad N \geq \bar{N}, \quad 2^{-2 J} \leq \delta \leq 2^{-(1.5) J}, \quad 1 \leq \beta \leq \bar{\beta} .
$$


For simplicity we keep these parameters implicit and omit them from the notations for $X_{I}(N, \delta, c, C), \Psi_{I}(N, \delta, C), M_{\omega}(\beta)$ and $\mathbb{S}_{t}(N, \delta, \beta, c, C)$. Therefore, the reader is warned that the precise values of these parameters may vary from line to line without explicit mention.

An advantageous example of usage for this convention is that whenever $\chi \in X_{I}$, the functions $\chi^{m} \in X_{I}$ as well for small values of $m \in \mathbb{N}$.

In our arguments we will make use of a form of Bernstein's inequality involving approximate indicators, in particular functions of the classes $X_{I}$ described above. This is a known phenomenon in the literature, see e.g. [28, Lemma 5.4]; we give the proof as we are in the vector-valued context.

2.4. Lemma. Let $R>0, \mathcal{X}$ be a Banach space and $f$ be an $\mathcal{X}$-valued function on $\mathbb{R}$ with

$$
\operatorname{supp} \widehat{f} \subset B_{R} \text {. }
$$

Let $w: \mathbb{R} \rightarrow(0, \infty)$ be essentially constant at scale $R^{-1}$, namely

$$
A^{-1}\langle R|x-y|\rangle^{-100} \leq \frac{w(x)}{w(y)} \leq A\langle R|x-y|\rangle^{100}, \quad x, y \in \mathbb{R}
$$

for some positive constant $A$. Then for all $0<\alpha \leq 1$

$$
\|w f\|_{L^{\infty}(\mathbb{R} ; X)} \lesssim_{A, \alpha} R^{\alpha}\|w f\|_{L^{\frac{1}{\alpha}(\mathbb{R} ; X)}} .
$$

Proof. Let $\phi$ be a smooth nonnegative function with $\phi=1$ on $B_{R}$ and $\phi=0$ on $B_{2 R}$. Notice that $|\widehat{\phi}(x)| \lesssim R\langle R|x|\rangle^{-200}$, for all $x \in \mathbb{R}$. Then $f=f * \widehat{\phi}$, and

$$
\begin{aligned}
|w(x) f(x)|_{X} & \lesssim \alpha_{, A} R \int \frac{|w(y) f(y)|_{X}}{\langle R|x-y|\rangle^{100}} \mathrm{~d} y \\
& \leq R^{\alpha}\|w f\|_{L^{\frac{1}{\alpha}(\mathbb{R} ; \mathcal{X})}}\left(\int \frac{R \mathrm{~d} y}{\langle R|x-y|\rangle^{100}}\right)^{1-\alpha} \lesssim R^{\alpha}\|w f\|_{L^{\frac{1}{\alpha}(\mathbb{R} ; \mathcal{X})}}
\end{aligned}
$$

as claimed. The proof is complete.

We will apply the lemma above to $w=\chi \in X_{I}$ for values $R \sim \ell(I)$.

2.3. Rank 1 forms. Trilinear multiplier forms of the type (1) admit a discretization in term of tri-tiles. We say that the ordered triple of tiles $P=\left(P_{1}, P_{2}, P_{3}\right)$ is a tri-tile if

$$
I_{P_{1}}=I_{P_{2}}=I_{P_{3}}=: I_{P} \text {. }
$$

Tri-tiles specify the space-frequency essential support of single scale multiplier forms, spatially concentrated on $I_{P}$ and frequency supported on the frequency cube

$$
Q_{P}=\omega_{P_{1}} \times \omega_{P_{2}} \times \omega_{P_{3}} .
$$

As condition (1) is invariant under the one-parameter family of translations along the vector $\beta^{\perp}$, the decomposition of the forms (1) is realized with a one-parameter, or rank 1 , family of tri-tiles, which is defined as follows.

Let $J>10$ be a (large) constant depending on $\Delta_{\beta}$. The collection of tri-tiles $\mathbb{P}$ is of rank 1 if the following hold.

a. The collections $\left\{I_{P}: P \in \mathbb{P}\right\}$ and $\left\{\omega_{P_{k}}: P \in \mathbb{P}\right\}, k=1,2,3$ are $O\left(2^{J}\right)$ scale-separated $J$-dyadic grids;

b. If $P \neq P^{\prime} \in \mathbb{P}$ are such that $I_{P}=I_{P^{\prime}}$ then $\omega_{P_{j}} \cap \omega_{P_{j}^{\prime}}=\varnothing$ for each $j \in\{1,2,3\}$; 
c. Denote by $\omega_{P}$ the convex hull of the intervals $3 \omega_{P_{k}}, k=1,2,3$. If $P, P^{\prime} \in \mathbb{P}$ are such that $\omega_{P_{k}} \subset \omega_{P_{k}^{\prime}}$ for some $k \in\{1,2,3\}$ then $2^{\frac{I}{2}} \omega_{P} \subset 2^{\frac{I}{2}} \omega_{P^{\prime}}$;

d. if $P, P^{\prime} \in \mathbb{P}$ are such that $\omega_{P_{j}} \subset \omega_{P_{j}^{\prime}}$ for some $j \in\{1,2,3\}$ then $3 \omega_{P_{k}} \cap 3 \omega_{P_{k}^{\prime}}=\varnothing$ for $k \in\{1,2,3\} \backslash\{j\}$.

The singular multiplier forms $\Lambda_{m}$ then lie in the convex hull of the tri-tile forms

$$
\Lambda_{\mathbb{P}}\left(f_{1}, f_{2}, f_{3}\right)=\sum_{P \in \mathbb{P}} \int_{\mathbb{R}} \prod_{k=1}^{3} \chi_{I_{P}}(x) T_{m_{P_{k}}} f_{k}(x) \mathrm{d} x
$$

where $\mathbb{P}$ is a finite subset of a rank 1 collection of tri-tiles, $\chi_{I_{P}} \in X_{I_{P}}$ has been defined in Subsection 2.2 and $m_{P_{k}} \in M_{\omega_{P_{k}}}(1), P \in \mathbb{P}$ satisfies the consistency condition

$$
Q_{P}=Q_{P^{\prime}} \Longrightarrow m_{P_{k}}=m_{P_{k^{\prime}}} k=1,2,3,
$$

referring to (2.3). Therefore, to bound $\Lambda_{m}$ it suffices to bound $\Lambda_{\mathbb{P}}$ uniformly. A detailed proof of these statements, in a more general context, is given in [27, Section 5], see also $[28,32]$. The remainder of the article will be devoted to the proof of such uniform bounds for $\Lambda_{\mathbb{P}}$. Note that the operators

$$
f \mapsto \chi_{I_{P}}\left(T_{m_{P_{k}}} f\right)
$$

belong to the class $\mathbb{S}_{P_{k}}$, for $k=1,2,3$.

\section{Proof of Theorem 1.1: tree and energy estimates}

In this section, after devising the necessary definitions in our context, we present the statements of the three main lemmas, which may then be combined to prove Theorem 1.1 in a standard fashion.

We first introduce trees, roughly speaking, collections of tri-tiles sitting at a common frequency and spatially localized to an interval. Then we define tree operators, that is modulated Calderón-Zygmund localized operators associated to each tree. These are used to define the energy of a certain $\mathcal{X}$-valued function with respect to a set of tri-tiles $\mathbb{P}$ : this is a sort of localized maximal $L^{q}(\mathbb{R} ; \mathcal{X})$-norm of tree operators coming from $\mathbb{P}$.

Finally, we state the main steps in the proof of Theorem 1.1. The first is the energy lemma, which allows us to decompose any given collection of tri-tiles into are unions of trees of controlled energy for each function $f_{k}$ and bounded spatial support. The second is the tree lemma, which provides a bound of the tri-tile form (2.3) when $\mathbb{P}$ is a tree. The proof of this lemma is one of the main novelties of this article, as it relies on a combination of the multilinear UMD CZ theory of [12] with newly developed phase-space projections adapted to the vector-valued setting.

We remark here that a standard combination of Lemmata 3.3, 3.2 and 3.1 yields a range of restricted weak type estimates for the forms (2.3): the elementary procedure is identical to that leading to [21, Corollary 9.2]. These estimates then entail Theorem 1.1 by standard multilinear restricted weak type interpolation, see e.g. [32]. This deduction is the same as that of [21, Theorem 9.3] from [21, Corollary 9.2]. We omit the details.

3.1. Trees. Rank 0 subcollections of tri-tiles $\mathbb{P}$, whose associated forms $\Lambda_{\mathbb{P}}$ are discretized multilinear CZ type multipliers, are called trees. We work with a specific notion of tree which satisfies certain additional properties along the lines of [28, Section 4]. We say that $\mathrm{T} \subset \mathbb{P}$ is a tree having $\left(I_{\mathrm{T}}, \xi_{\mathrm{T}}\right)$ as top data if the following conditions hold. 
a. $I_{\top}$ is a $J$-dyadic interval, $\xi_{\top} \in \mathbb{R}$ and

$$
I_{P} \subset I_{\mathrm{T}}, \quad \xi_{\mathrm{T}} \in \omega_{P} \quad \forall P \in \mathrm{T} .
$$

b. The frequency localization sets $\mathbf{Q}_{\boldsymbol{T}}=\left\{Q_{P}: P \in \mathrm{T}\right\}$ is such that

$$
Q, Q^{\prime} \in Q_{\top}, \ell(Q)=\ell\left(Q^{\prime}\right) \Longrightarrow Q=Q^{\prime} ;
$$

namely, there is only one frequency localization for each $J$-dyadic scale.

c. The spatial localization sets

$$
E_{Q, T}=\bigcup\left\{I_{P}: P \in \mathrm{T}: Q_{P}=Q\right\}, \quad Q \in \mathbf{Q}_{\mathbf{T}}
$$

are nested, that is

$$
Q, Q^{\prime} \in Q_{\top}, \ell(Q) \leq \ell\left(Q^{\prime}\right) \Longrightarrow E_{Q, T} \supset E_{Q^{\prime}, T} .
$$

It is convenient to denote by $\mathbf{j}_{\top}=\left\{j \in \mathbb{Z}: \ell(Q)=2^{j J}\right.$ for some $\left.Q \in \mathbf{Q}_{\top}\right\}$, the frequency scales appearing in $\mathrm{T}$. Then

$$
\mathrm{T}=\bigcup_{j \in \mathbf{j}_{\boldsymbol{\top}}} \mathrm{T}(j), \quad \mathrm{T}(j)=\left\{P \in \mathrm{T}: \ell\left(Q_{P}\right)=2^{j J}\right\} .
$$

We also take the opportunity here to observe that trees constructed via greedy selection processes, such as the one in the proof of Lemma 3.2 below, satisfy properties $b$. and c. automatically. This is proved in e.g. [28, Lemmata 4.4 and 4.7]. Furthermore, the smoothing properties of [28, Lemmata $4.10,4.11,4.12]$ also hold. We will only make use of these properties within the proof of the phase-space projection estimates: we will then recall what is needed, and send to [28] for detailed statements.

3.2. Tree operators. We say that the tree $\mathrm{T}$ is $k$-lacunary, $k=1,2,3$ if

$$
\left\{3 \omega_{P_{k}}: P \in \mathrm{T}_{A}\right\} \text { are a pairwise disjoint collection for } k \in A .
$$

A consequence of property $\mathrm{d}$. of rank 1 collections is that each tree $\mathrm{T}$ can be written as the disjoint union

$$
\mathrm{T}=\bigcup_{\substack{A \subset\{1,2,3\} \\ \# A \geq 2}} \mathrm{~T}_{A}
$$

where each $\mathrm{T}_{A}$ is a tree with the same top data as $\mathrm{T}$ and has the additional property (3.2) for $k \in A$.

We now introduce tree operators associated to $k$-lacunary trees. For our purposes here, we need a more nuanced notion than the usual, e.g. appearing in $[9,19,25,26]$, fully discretized tree operator

$$
f \mapsto \sum_{P \in \mathrm{T}}\left\langle f, \varphi_{P_{k}}\right\rangle \varphi_{P_{k}}, \quad k \in A
$$

where $\varphi_{P_{k}}$ is a wave packet adapted to the tile $P_{k}$. Let T be a $k$-lacunary tree. A (scalar) tree operator of $k$-th type is the linear operator

$$
T_{\mathrm{T}} f=\sum_{P \in \mathrm{T}} S_{P_{k}} f
$$


where each $S_{P_{k}} \in \mathbb{S}_{P_{k}}, P \in \mathrm{T}$. When $\xi_{\mathrm{T}}=0$, the defined tree operator is a pseudodifferential operator with symbol

$$
a(x, \xi)=\sum_{P \in \mathbf{T}} \psi_{P_{k}}(x) m_{P_{k}}(\xi)
$$

where each $\psi_{P_{k}} \in \Psi_{I_{P}}$ and $m_{P_{k}} \in M_{\omega_{P_{k}}}$. A routine computation verifies that this symbol is uniformly of class $S_{1,1}^{0}$, see e.g. [28, p. 288]. Further, as the intervals $\left\{\omega_{P_{k}}: P \in \mathrm{T}\right\}$ are pairwise disjoint, $T_{\mathrm{T}}$ is uniformly $L^{2}(\mathbb{R})$ bounded. Relying on these two observations, we gather that $T_{\mathrm{T}}$ is an $L^{2}(\mathbb{R})$-bounded Calderón-Zygmund operator, see for instance the discussion at [31, p. 271]. Therefore, $T_{\mathrm{T}}$ satisfies uniform $L^{q}(\mathbb{R} ; \mathcal{X})$ bounds, $1<q<\infty$, as well $L^{\infty}(\mathbb{R} ; \mathcal{X}) \rightarrow \mathrm{BMO}(\mathbb{R} ; \mathcal{X})$ estimates, whenever $\mathcal{X}$ is a UMD Banach space. In fact, by modulation invariance, we may remove the $\xi_{\mathrm{T}}=0$ assumption and conclude that tree operators $T_{\mathrm{T}}$ are uniformy $L^{q}(\mathbb{R} ; \mathcal{X})$ bounded, when $1<q<\infty$.

3.3. Energy and energy lemma. This definition is instead a re-elaboration of $[19$, Section 8]. If $q \geq 2, \mathrm{~T}$ is a $k$-lacunary tree and $f$ is a $\mathcal{X}$-valued function, we define

$$
\|f\|_{T, k, q}=\sup \frac{1}{\left|I_{\top}\right|^{\frac{1}{q}}}\left\|T_{\mathrm{T}} f\right\|_{L^{q}(\mathbb{R} ; X)}
$$

where the sup is taken over all possible choices of type $k$ tree operators $T_{\mathrm{T}}$. We also find convenient to define a maximal version: for each set $\mathbb{P}$ of tri-tiles,

$$
\text { eng }_{k}(f)(\mathbb{P} ; q)=\sup _{\substack{\mathrm{T} \subset \mathbb{P} \\ \mathrm{T} k \text {-lacunary }}}\|f\|_{\mathrm{T}, k, q} \text {. }
$$

The next lemma is a variation of e.g. [19, Corollary 9.6].

3.5. Lemma. Let $\mathbb{P}$ be a finite collection of tri-tiles. Then

$$
\operatorname{eng}_{k}(f)(\mathbb{P} ; q) \lesssim \sup _{P \in \mathbb{P}} \inf _{I_{P}} \mathrm{M}\left(|f|_{\mathcal{X}}\right) .
$$

Although the arguments of [19] may be adapted to the context of Lemma 3.1, we provide a more direct proof in Section 7.

In the last main lemma, the quantitative assumption of $\mathcal{X}_{k}$ being an interpolation space is used. We could alternatively bring forth definitions akin to the tile-type of a Banach space in $[19,21,22]$, which is a formal consequence of our intermediate UMD assumption, but for simplicity and lack of examples we give up on this additional formal generality.

3.6. Lemma. Suppose $\mathcal{X}$ is $q_{\mathcal{X}}$-intermediate and let $f \in L^{\infty}(\mathbb{R} ; \mathcal{X})$ be subordinated to the finite measure set $F$, namely $|f|_{X} \leq \mathbf{1}_{F}$. Fix $q>q_{X}$ and let $\mathbb{P}$ be a finite set of tri-tiles. Then $\mathbb{P}=\mathbb{P}^{+} \cup \mathbb{P}^{-}$ with the property that

$$
\operatorname{eng}_{k}(f)\left(\mathbb{P}^{-} ; q_{X}\right) \leq 2^{-1} \text { eng }_{k}(f)\left(\mathbb{P} ; q_{X}\right)
$$

and that $\mathbb{P}^{+}$is a union of trees $T \in \mathcal{T}$ with the property that

$$
\sum_{T \in \mathcal{T}}\left|I_{\top}\right| \lesssim_{q}\left[\operatorname{eng}_{k}(f)\left(\mathbb{P} ; q_{X}\right)\right]^{-q}|F| .
$$

The proof of Lemma 3.2 is a revisitation of the steps leading to [19, Proposition 8.4] and is postponed to Section 6. Note that Lemma 3.2 is the only main step of the proof of Theorem 1.1 where a $q_{\mathcal{X}}$-intermediate assumption is used. 
The final main tool of the proof of Theorem 1.1 is a bound on the forms (2.3) in terms of energy parameters in the particular case where the collection $\mathbb{P}$ is a tree.

3.7. Lemma. Let $\mathcal{X}_{k}, k=1,2,3$ be UMD spaces and

$$
2 \leq q_{1}, q_{2}, q_{3}<\infty, \quad \sum_{k=1}^{3} \frac{1}{q_{k}} \geq 1 .
$$

Let $\mathrm{T}$ be a tree. With reference to (2.3) there holds

$$
\left|\Lambda_{\mathrm{T}}\left(f_{1}, f_{2}, f_{3}\right)\right| \lesssim\left|I_{\mathrm{T}}\right| \prod_{k=1}^{3} \mathrm{eng}_{k}\left(f_{k}\right)\left(\mathrm{T} ; q_{k}\right) .
$$

The proof of Lemma 3.3 uses a novel vector-valued version of the phase-space projection technique of $[10,28]$ in conjunction with [12, Theorem 1.2] and is given in Section 4 .

\section{Phase space projections and the proof of the tree lemma}

We develop phase-space projections in the vector-valued context and combine them with the bounds for vector-valued extensions of bilinear CZ kernels to prove Lemma 3.3. The following treatment is an adaptation of the construction made in [28, Sec. 7 and 8]. Our arguments are more involved due to the vector-valued nature of the involved functions. However, we take advantage of a significant simplification in that no uniformity issues are considered: in the language of [28] the indices $\mathbf{m}_{i}$ are all zero. Uniform estimates in the vector-valued context will be the object of future work.

In the main proposition of this section, we make use of Littlewood-Paley projections as follows. The operator $T_{j}$ is meant to be a Fourier multiplier whose symbol is real, even, supported on $\left[-2^{J(j+2)}, 2^{J(j+2)}\right]$ and equals one on $\left[-2^{J(j+1)}, 2^{J(j+1)}\right]$ and $S_{j}=T_{j}-T_{j-1}$. The projections $S_{j}$ appear also in Lemma 4.3 below.

4.1. Proposition (phase-space projections). For $k=1,2,3$, let $X_{k}$ be a UMD space and $q_{k} \in[2, \infty)$. Let $\mathrm{T}$ be a tree with the following properties:

i. $\xi_{\mathrm{T}}=0$;

ii. $T$ is lacunary in the components $k \in A$ and overlapping in the components $k \in B$, with $A \cup B=\{1,2,3\}$ disjoint union and $\# A \in\{2,3\}$;

iii. the separation of scales condition

$$
\inf \left\{\left|j-j^{\prime}\right|: j, j^{\prime} \in \mathbf{j} \mathbf{T}, j \neq j^{\prime}\right\} \geq M
$$

holds for some large constant $M$.

Choose $\left\{S_{P_{k}} \in \mathbb{S}_{P_{k}}, P \in \mathrm{T}, k \in\{1,2,3\}\right\}$. Then there are linear operators $\Pi_{k}$ with the following properties.

a. If $p \geq q_{k}$, there holds

$$
\left\|\Pi_{k} f\right\|_{L^{p}\left(\mathbb{R} ; X_{k}\right)} \lesssim\left|I_{T}\right|^{\frac{1}{p}} \operatorname{eng}_{k}(f)\left(\mathrm{T} ; q_{k}\right) ;
$$

b. if $k \in A$, for all $j \in \mathbf{j}_{\mathbf{T}}$, referring to (3.1)

$$
\sum_{P \in \mathrm{T}(j)} S_{P_{k}} f=S_{j}\left(\Pi_{k} f\right)
$$


c. If $p \geq q_{k}, k \in B, j_{0} \in \mathbf{j}_{\mathbf{\top}}$ and $\ell\left(I_{0}\right)=2^{-J j_{0}}$,

$$
\left\|\mathbf{1}_{I_{0}} \sum_{P \in \mathrm{T}\left(j_{0}\right)} S_{P_{k}}\left(f-\Pi_{k} f\right)\right\|_{L^{p}\left(\mathbb{R} ; X_{k}\right)} \lesssim\left|I_{0}\right|^{\frac{1}{p}} \operatorname{eng}_{k}(f)\left(\mathrm{T} ; q_{k}\right) \int_{\mathbb{R}} \chi_{I_{0}}(x) \mu_{j_{0}}(x) \frac{\mathrm{d} x}{\left|I_{0}\right|} .
$$

where $\mu_{j}$ is as in [28, eq. (51)]. Furthermore

$$
\left\|\mathbf{1}_{I_{0}} \sum_{P \in \mathrm{T}\left(j_{0}\right)} S_{P_{k}} \Pi_{k} f\right\|_{L^{p}\left(\mathbb{R} ; \mathcal{X}_{k}\right)} \lesssim\left|I_{0}\right|^{\frac{1}{p}} \text { eng }_{k}(f)\left(\mathrm{T} ; q_{k}\right) .
$$

While the operators $\Pi_{k}$ depend on the choice of $\left\{S_{P_{k}} \in \mathbb{S}_{P_{k}}, P \in T, k \in\{1,2,3\}\right\}$, the estimates above are uniform over such choice.

The proof of Proposition 4.1 is postponed to the next section. Herein, we proceed to showing how such proposition may be coupled with the main result of [12] to obtain the tree estimate we claimed in Lemma 3.3. The next subsection contains some preliminaries while the main line of argument is given in Subsection 4.2

4.1. Preliminaries. We begin with a preliminary localized single scale estimate for tree operators which will be of use towards Lemma 3.3 as well as in Section 5.

4.5. Lemma. Let $I_{0}$ be a J-dyadic interval with $\ell\left(I_{0}\right)=2^{-j_{0} J}, \psi_{I_{0}} \in \Psi_{I_{0}}, S_{P_{k}} \in \mathbb{S}_{P_{k}}$ for each $P \in \mathrm{T}\left(j_{0}\right)$. Then

$$
\left\|\psi_{I_{0}} \sum_{P \in \mathrm{T}\left(j_{0}\right)} S_{P_{k}} f\right\|_{L^{p}\left(\mathbb{R} ; X_{k}\right)} \lesssim\left|I_{0}\right|^{\frac{1}{p}} \operatorname{eng}_{k}(f)\left(\mathrm{T} ; q_{k}\right), \quad q_{k} \leq p \leq \infty .
$$

Arguing by interpolation, it suffices to prove the extremal cases.

Proof of (4.2) for $p=q_{k}$. Let $n \in \mathbb{N}$. There are at most two $P \in \mathrm{T}\left(j_{0}\right)$ such that $\operatorname{dist}\left(I_{P}, I_{0}\right)=$ $n 2^{-j_{0} J}$. Fix such a $P$. It then suffices to estimate

$$
\left\|\psi_{I_{0}} S_{P_{k}} f\right\|_{L^{q_{k}\left(\mathbb{R} ; X_{k}\right)}} \lesssim\langle n\rangle^{-100}\left|I_{0}\right|^{\frac{1}{q_{k}}} \text { eng }_{k}(f)\left(\mathrm{T} ; q_{k}\right) .
$$

Write $S_{P_{k}} f=\psi_{P} T_{m_{P_{k}}} f$. Then $\widetilde{\psi}:=\langle n\rangle^{100} \psi_{I_{0}} \psi_{P} \in \Psi_{I_{P}}$ and the estimate in the last display simply follows from the definition of $\mathrm{eng}_{k}(f)\left(\mathrm{T} ; q_{k}\right)$.

Proof of (4.2) for $p=\infty$. The function we are estimating has frequency support in a ball of radius $O\left(2^{j_{0} J}\right)$. Then this case follows from the case $p=q_{k}$ and a straightforward application of Lemma 2.2.

We then particularize the definition (2.3) to the case where $\mathbb{P}$ is our tree $T$. By modulation, translation and scaling invariance, we may reduce Lemma 3.3 to the case $\xi_{\mathrm{T}}=0, I_{\mathrm{T}}=[0,1)$. Notice that, referring to (2.3), (3.1), we have $Q_{P}=Q_{P^{\prime}}:=Q_{j}$ for all $P, P^{\prime} \in \mathrm{T}(j)$, and that consequently $m_{P_{k}}=m_{P_{k}^{\prime}}:=m_{j, k}$ for all $P, P^{\prime} \in \mathrm{T}(j), k=1,2,3$. Therefore we may set for $j \in \mathbf{j}_{\boldsymbol{T}}$

$$
\begin{aligned}
& \tilde{\chi}_{j}:=\sum_{P \in \mathrm{T}(j)} \chi_{I_{P}}^{3} \\
& \tilde{\pi}_{j, k}:=T_{m_{j, k},} \quad k=1,2,3
\end{aligned}
$$


and rewrite, and subsequently estimate, (2.3) for $\mathrm{T}=\mathbb{P}$ as, cf. [28, eq. (44)]

$$
\left|\Lambda_{\mathbf{T}}\left(f_{1}, f_{2}, f_{3}\right)\right|=\left|\sum_{j \in \mathbf{j} \mathbf{T}} \int_{\mathbb{R}} \tilde{\chi}_{j} \prod_{k=1}^{3} \tilde{\pi}_{j, k} f_{k}\right| \leq \sum_{j \in \mathbf{j} \mathbf{T}}\left|\int_{\mathbb{R}} \tilde{\chi}_{j} \prod_{k=1}^{3} \tilde{\pi}_{j, k} f_{k}\right| .
$$

An analogous argument to the one that proves [28, Lemma 7.3], provided that the vectorvalued Lemma 4.2 is used in place of [28, Lemma 7.2], yields that

$$
\sum_{j \in \mathbf{j}_{\mathbf{T}}}\left|\int_{\mathbb{R}} \tilde{\chi}_{j}(x) \prod_{k=1}^{3} \tilde{\pi}_{j, k} f_{k}(x)-\prod_{k=1}^{3} \tilde{\chi}_{j}(x) \tilde{\pi}_{j, k} f_{k}(x) \mathrm{d} x\right| \lesssim \prod_{k=1}^{3} \text { eng }_{k}\left(f_{k}\right)\left(\mathrm{T} ; q_{k}\right)
$$

which is acceptable for the estimate of Lemma 3.3. Therefore we have reduced the Lemma to proving that

$$
\left|\sum_{j \in \mathbf{j}_{\mathbf{T}}} \varepsilon_{j} \int_{\mathbb{R}} \prod_{k=1}^{3} \tilde{\chi}_{j} \tilde{\pi}_{j, k} f_{k}\right| \lesssim \prod_{k=1}^{3} \text { eng }_{k}\left(f_{k}\right)\left(\mathrm{T} ; q_{k}\right)
$$

uniformly over choices of unimodular coefficients $\left\{\varepsilon_{j}: j \in \mathbf{j}_{\mathbf{T}}\right\}$ which is the core of the argument, and is left for the next subsection.

The final preliminary result is a Hölder type estimate for the vector-valued extension of a classical trilinear paraproduct form. Such estimate is a particular case of the main result of [12] and depends only on the UMD property of the spaces involved.

4.8. Lemma. Let $\left\{p_{k}: k=1,2,3\right\}$ be a Hölder tuple of exponents with $1<p_{k}<\infty$ for all $k=1,2,3$. Let $\mathcal{X}_{k}$ be UMD spaces with a trilinear contraction $\prod_{k=1}^{3} \mathcal{X}_{k} \rightarrow \mathbb{C}$. Let $g_{k} \in$ $\left(L^{1}(\mathbb{R}) \cap L^{\infty}(\mathbb{R})\right) \otimes \mathcal{X}_{k}$, for $k=1,2,3$. Then

$$
\left|\int \sum_{j \in \mathbf{j} \boldsymbol{\top}} \varepsilon_{j}\left(\tilde{\pi}_{j, 1} g_{1}\right)\left(S_{j} g_{2}\right)\left(S_{j} g_{3}\right)\right| \lesssim \prod_{k=1}^{3}\left\|g_{k}\right\|_{L^{p_{k}}\left(\mathbb{R} ; \boldsymbol{X}_{k}\right)} .
$$

Proof. Recall that $m_{j, 1}$, the symbol of $\tilde{\pi}_{j, 1}$, is adapted and supported in a moderate dilate of $Q_{j, 1}$, which is a dyadic interval of length $2^{j J}$ and such that $2^{\frac{I}{2}} Q_{j, 1}$ contains the origin. Thus $m_{j, 1}$ vanishes outside $|\xi| \leq 2^{J\left(j+\frac{1}{2}\right)}$. The symbol $\Psi_{j}$ of $S_{j}$ is supported on $2^{J(j+1)} \leq|\xi| \leq 2^{J(j+2)}$. Let $g_{k} \in L^{1}(\mathbb{R}) \cap L^{\infty}(\mathbb{R})$ be scalar functions. Then Plancherel's equality yields

$$
\sum_{j \in \mathbf{j} \mathbf{T}} \varepsilon_{j}\left(\tilde{\pi}_{j, 1} g_{1}\right)\left(S_{j} g_{2}\right)\left(S_{j} g_{3}\right)=\left\langle O\left(g_{1}, g_{2}\right), \overline{g_{3}}\right\rangle,
$$

where $O$ is the bilinear Fourier multiplier operator

$$
\begin{aligned}
& O\left(g_{1}, g_{2}\right)(x)=\int_{\mathbb{R} \times \mathbb{R}} \widehat{g_{1}}(\xi) \widehat{g_{2}}(\xi) m\left(\xi_{1}, \xi_{2}\right) e^{2 \pi i x\left(\xi_{1}+\xi_{2}\right)} \mathrm{d} \xi_{1} \mathrm{~d} \xi_{2}, \quad x \in \mathbb{R}, \\
& m\left(\xi_{1}, \xi_{2}\right):=\sum_{j \in \mathbf{j} \boldsymbol{T}} \varepsilon_{j} m_{j, 1}\left(\xi_{1}\right) \Psi_{j}\left(\xi_{2}\right) \Psi_{j}\left(-\xi_{1}-\xi_{2}\right) .
\end{aligned}
$$

The support and smoothness conditions on $m_{j, 1}, \Psi_{j}$ imply that $m$ satisfies the CoifmanMeyer condition multiplier and thus $O$ is a bilinear $C Z$ kernel operator. We may then use 
[12, Theorem 1.2] to conclude that $O$ extends to a bounded bilinear operator

$$
L^{p_{1}}\left(\mathbb{R} ; \mathcal{X}_{1}\right) \times L^{p_{2}}\left(\mathbb{R} ; \mathcal{X}_{2}\right) \rightarrow L^{p_{3}^{\prime}}\left(\mathbb{R} ; \mathcal{X}_{3}^{\prime}\right) .
$$

As (4.1) continues to hold for $g_{k} \in\left(L^{1}(\mathbb{R}) \cap L^{\infty}(\mathbb{R})\right) \otimes \mathcal{X}_{k}$, the vector-valued bound of the above display and duality complete the proof of the lemma.

4.2. Proof of Lemma 3.3, estimate (4.1). By the condition (3.3), we may find a Hölder tuple $p_{1}, p_{2}, p_{3}$ with $q_{k} \leq p_{k}<\infty$. To apply Proposition 4.1 it is useful to keep in mind the equalities

$$
\int_{\mathbb{R}} \prod_{k=1}^{3} \tilde{\chi}_{j} \tilde{\pi}_{j, k} f_{k}=\int_{\mathbb{R}} \prod_{k=1}^{3}\left(\sum_{P \in \mathrm{T}(j)} S_{P_{k}} f_{k}\right), \quad j \in \mathbf{j}_{\mathbf{T}}
$$

having called $S_{P_{k}} \in \mathbb{S}_{P_{k}}$ the operator $f \mapsto \chi_{I_{P}} T_{m_{j, k}} f_{k}$. We first handle the easy case where $B=\varnothing$. Applying part b. of Proposition 4.1 to each $f_{k}$, for each $j \in \mathbf{j}_{\boldsymbol{\top}}$, we have

$$
\sum_{j \in \mathbf{j} \boldsymbol{\top}} \varepsilon_{j} \int_{\mathbb{R}} \prod_{k=1}^{3} \tilde{\chi}_{j} \tilde{\pi}_{j, k} f_{k}=\sum_{j \in \mathbf{j} \boldsymbol{T}} \varepsilon_{j} \int_{\mathbb{R}} \prod_{k=1}^{3} S_{j}\left(\Pi_{k} f_{k}\right) .
$$

For $k=1,2,3$ let $\sigma_{k}=\left\{\sigma_{j, k}: j \in \mathbf{j}_{\top}\right\}$ be a sequence of i.i.d. random variables which take the values $1,-1$ with equal probability. We denote the expectation with respect to $\sigma_{k}$ by $\mathbb{E}^{k}$.

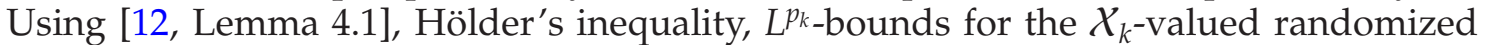
square function (this holds since $\mathcal{X}_{k}$ is UMD), and subsequently part a. of Proposition 4.1, there holds

$$
\begin{aligned}
& \left|\sum_{j \in \mathbf{j} \boldsymbol{T}} \varepsilon_{j} \int_{\mathbb{R}} \prod_{k=1}^{3} S_{j}\left(\Pi_{k} f_{k}\right)\right| \\
& \left.\lesssim \prod_{k=1}^{3}\left(\mathbb{E}^{k} \int_{\mathbb{R}}\left|\sum_{j \in \mathbf{j}_{\boldsymbol{\top}}} \sigma_{j, k} S_{j}\left(\Pi_{k} f_{k}\right)\right|_{\mathcal{X}_{k}}^{p_{k}}\right)^{\frac{1}{p_{k}}} \lesssim \prod_{k=1}^{3} \| \Pi_{k} f_{k}\right) \|_{L^{p_{k}}\left(\mathbb{R} ; \boldsymbol{X}_{k}\right)} \lesssim \prod_{k=1}^{3} \text { eng }_{k}\left(f_{k}\right)\left(\mathrm{T} ; q_{k}\right),
\end{aligned}
$$

which is the claim (4.1).

We turn to the harder case where $\# A=2$. By symmetry we may work with $B=\{1\}$. We use Proposition 4.1 to bound the left hand side of (4.1) by MAIN $+E R R_{1}+E R R_{2}$ where

$$
\begin{aligned}
& \text { MAIN }:=\left|\sum_{j \in \mathbf{j} \boldsymbol{T}} \varepsilon_{j} \int_{\mathbb{R}} \tilde{\pi}_{j, 1}\left(\Pi_{1} f_{1}\right) \prod_{k=2}^{3} \tilde{\chi}_{j} \tilde{\pi}_{j, k} f_{k}\right| \\
& \mathrm{ERR}_{1}:=\sum_{j \in \mathbf{j} \boldsymbol{\top}} \sum_{\ell(I)=2^{-j J}} \int_{I}\left|\tilde{\chi}_{j} \tilde{\pi}_{j, 1}\left(f_{1}-\Pi_{1} f_{1}\right)\right|_{X_{1}} \prod_{k=2}^{3}\left|\tilde{\chi}_{j} \tilde{\pi}_{j, k} f_{k}\right|_{\mathcal{X}_{k}} \\
& \mathrm{ERR}_{2}:=\sum_{j \in \mathbf{j} \boldsymbol{j}} \sum_{\ell(I)=2^{-j J}} \int_{I} \zeta_{j}\left|\tilde{\pi}_{j, 1}\left(\Pi_{1} f_{1}\right)\right|_{X_{1}} \prod_{k=2}^{3}\left|\left(\tilde{\chi}_{j}\right)^{\frac{1}{6}} \tilde{\pi}_{j, k} f_{k}\right|_{\mathcal{X}_{k}}, \quad \zeta_{j}:=\left|\left(\tilde{\chi}_{j}^{2}-\tilde{\chi}_{j}^{3}\right)\right|\left(\tilde{\chi}_{j}\right)^{\frac{1}{2}}
\end{aligned}
$$

the second and third of which are error terms. In $\mathrm{ERR}_{j}$ the sum over $I$ is over all $J$-dyadic intervals of a fixed length $2^{-j J}$. 
We first handle the error terms: via Hölder's inequality with exponents $p_{k}$, and a combination of (4.1) for the $\mathcal{X}_{1}$ with Lemma 4.2 for the $\mathcal{X}_{k}$ factors, $k=2$, 3 , we achieve the estimates

$$
\mathrm{ERR}_{1} \lesssim\left(\prod_{k=1}^{3} \text { eng }_{k}\left(f_{k}\right)\left(\mathrm{T} ; q_{k}\right)\right) \sum_{j \in \mathbf{j} \mathbf{T}} \sum_{\ell(I)=2^{-j J}} \int x_{I} \mu_{j} \lesssim\left(\prod_{k=1}^{3} \text { eng }_{k}\left(f_{k}\right)\left(\mathrm{T} ; q_{k}\right)\right) \sum_{j \in \mathbf{j} \mathbf{T}} \int \mu_{j} .
$$

As detailed in [28, p.286, Lemma 4.12] the $j$-summation above is $\lesssim\left|I_{\top}\right|=1$, showing that $E R R_{1}$ complies with the right hand side of (4.1). The second error term is bounded also using Hölder, followed by the single scale estimates (4.1) and Lemma 4.2:

$$
\mathrm{ERR}_{2} \lesssim\left(\prod_{k=1}^{3} \text { eng }_{k}\left(f_{k}\right)\left(\mathrm{T} ; q_{k}\right)\right) \sum_{j \in \mathbf{j}_{\mathbf{T}}} \sum_{\ell(I)=2^{-j I}}|I|\left\|\mathbf{1}_{I} \zeta_{j}\right\|_{\infty} .
$$

The $j, I$ summation above is also bounded by $\left|I_{T}\right|$ via $[28$, Lemma 4.8]. We omit the details.

We move to the main term. Using part b. of the Proposition, we recognize that

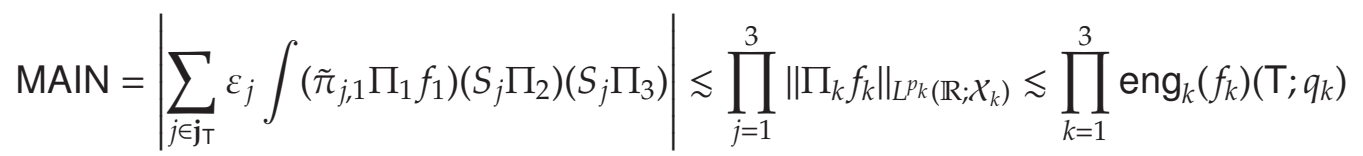

having used Lemma 4.3 for the first bound, and (4.1) for the second. This completes the proof of Lemma 3.3.

\section{Proof of Proposition 4.1}

In all cases below, the index $k \in\{1,2,3\}$ is fixed and we avoid mentioning it whenever possible. For instance, we write $q$ for $q_{k}, \mathcal{X}$ for $\mathcal{X}_{k}$ and $\alpha=1-\frac{1}{q}$.

5.1. Proof of Proposition 4.1, a. and b. parts: lacunary case. We recall that each $S_{P_{k}} f(x)=$ $\chi_{P} T_{m_{P}}$ with $\chi_{P} \in X_{I_{P}}$ and $m_{P} \in M_{\omega_{P_{k}}}$. Preliminarily observe that due to the support conditions on $m_{P}$ and $\widehat{\chi_{P}}$, the Fourier transforms of the functions

$$
\Pi_{k, j} f=\sum_{P \in \mathrm{T}(j)} S_{P_{k}} f
$$

are supported in the disjoint intervals $\left\{\xi: 2^{j J-1} \leq|\xi| \leq 2^{j J+1}\right\}$ where the symbol of $S_{j}$ is constant equal to one. In the lacunary case, the definition of $\Pi_{k}$ is then very simple, namely referring to (5.1)

$$
\Pi_{k}:=\sum_{j \in \mathbf{J}_{\mathbf{\top}}} \Pi_{k, j}
$$

and the equality in $b$. is immediate from the above considerations, while the estimate in a. for $p=q_{k}$ is immediate from the definition, as $\Pi_{k}$ is itself a tree operator. We now prove the estimate

$$
\left\|\Pi_{k} f\right\|_{\mathrm{BMO}\left(\mathbb{R} ; \mathcal{X}_{k}\right)} \lesssim \operatorname{eng}_{k}(f)\left(\mathrm{T} ; q_{k}\right)
$$

and a. for the other values of $p$ will follow by interpolation. 
Fix a $J$-dyadic interval $I$. We first bound the contribution of the large scales: set $\mathrm{T}^{+}=\left\{P \in \mathrm{T}: \ell\left(I_{P}\right)>\ell(I)\right\}$. Then if $P \in \mathrm{T}^{+}$with $\ell\left(I_{P}\right)=2^{v} \ell(I)$ and $\operatorname{dist}\left(I_{P}, I\right) \sim 2^{n} \ell\left(I_{P}\right)$, $v, n \in \mathbb{N}$, the Poincarè inequality yields

$$
\operatorname{osc}_{I}\left(S_{P_{k}} f\right) \lesssim \frac{1}{|I|^{\frac{1}{q}}}\left\|\ell(I) \nabla S_{P_{k}} f\right\|_{L^{q}(I ; \mathcal{X})} \leq 2^{-\alpha v} \frac{1}{\left|I_{P}\right|^{\frac{1}{q}}}\left\|\ell\left(I_{P}\right) \nabla\left(S_{P_{k}} f\right)\right\|_{L^{q}(I ; \mathcal{X})} .
$$

We write $\widetilde{m}_{P}(\xi):=\ell\left(I_{P}\right) \xi m_{P}(\xi)$, so that

$$
\begin{aligned}
\left|\ell\left(I_{P}\right) \nabla\left(S_{P_{k}} f\right)\right|_{X} & =\left|\chi_{P} T_{\widetilde{m}_{P}} f+\left(\ell\left(I_{P}\right) \nabla \chi_{I_{P}}\right) T_{m_{P}} f\right|_{X} \leq \widetilde{\chi_{I_{P}}}\left(\left|\widetilde{\chi_{I_{P}}} T_{\widetilde{m}_{P}} f\right|_{\mathcal{X}}+\left|\widetilde{\chi_{I_{P}}} T_{m_{P}} f\right|_{\mathcal{X}}\right) \\
& :=\widetilde{\chi_{I_{P}}}\left(\left|S_{P_{k}, 1} f\right|_{\mathcal{X}}+\left|S_{P_{k}, 2} f\right|_{X}\right)
\end{aligned}
$$

for a suitable choice of $\widetilde{\chi_{I_{P}}} \in X_{I_{P}}$ so that the domination of the last display holds. Observe that with this choice $S_{P_{k}, u}, u=1,2$, belong to the class $\mathbb{S}_{P_{k}}$ and are thus single scale tree operators, whence

$$
\frac{1}{\left|I_{P}\right|^{\frac{1}{q}}}\left\|S_{P_{k}, u}\right\|_{L^{q(\mathbb{R} ; X)}} \lesssim \operatorname{eng}_{k}(f)(\mathrm{T} ; q)
$$

Using the bounds $\left\|\widetilde{\chi_{P}}\right\|_{L^{\infty}(I)} \lesssim 2^{-100 n}$, we have proved that

$$
\operatorname{osc}_{I}\left(S_{P_{k}} f\right) \lesssim 2^{-\alpha v-100 n} \operatorname{eng}_{k}(f)(\mathrm{T} ; q) \text {. }
$$

which is summable over $P \in \mathrm{T}^{+}$, that is over $v, n \in \mathbb{N}$ as claimed.

We move to handling the small scales, that is $\mathrm{T}^{-}=\left\{P \in \mathrm{T}: \ell\left(I_{P}\right) \leq \ell(I)\right\}$. We may partition $\mathrm{T}^{-}$as the union of $\mathrm{T}^{-, 0}=\left\{P \in \mathrm{T}^{-}: I_{P} \subset 3 I\right\}$ and $\mathrm{T}^{-, n}=\left\{P \in \mathrm{T}^{-}: I_{P} \subset\right.$ $\left.\left(2^{n+1}+1\right) I \backslash\left(2^{n}+1\right) I\right\}$ for $n \geq 1$. We may choose $\widetilde{\chi}_{I} \in X_{I}$ so that the estimate

$$
\operatorname{osc}_{I}\left(\sum_{P \in \mathrm{T}^{-}} S_{P_{k}} f\right) \leq \frac{1}{|I|^{\frac{1}{q}}} \sum_{n \geq 0}\left\|g_{n}\right\|_{L^{q}(\mathbb{R} ; \mathcal{X})} \quad g_{n}=\sum_{P \in \mathrm{T}^{-, n}} \widetilde{\chi}_{I} S_{P_{k}} f
$$

holds. We now estimate each term appearing in the last summation over $n$. Fix $P \in \mathrm{T}^{-, n}$ for a moment and notice that $\operatorname{dist}\left(I, I_{P}\right) \sim 2^{n} \ell(I)$. Writing $S_{P_{k}} f=\chi_{I_{P}} T_{m_{P_{k}}} f$, define

$$
\zeta_{P}:=2^{100 n}{\widetilde{\chi_{I}}}_{\chi_{I_{P}}}, \quad \widetilde{S_{P_{k}}} f=\zeta_{P} T_{m_{P_{k}}} f .
$$

We claim that the function $\zeta_{P}$ belongs to $X_{I_{P}}$. Indeed, the decay condition (2.2) for $\zeta_{P}$ is easy to verify, with the additional $2^{100 n}$ factor being allowed by virtue of the previously observed separation between $I, I_{P}$. The frequency support condition (2.2) for $\zeta_{P}$ derives from the fact that the Fourier support of $\widetilde{\chi_{I}}$ has an equal or smaller scale than the Fourier support of $\widehat{\chi_{I_{p}}}$. Then we notice that $\mathrm{T}^{-, n}$ is a tree with top data $\left(I^{n}, 0\right)$ and contained in $\mathrm{T}$, whence

$$
\left\|g_{n}\right\|_{L^{q}(\mathbb{R} ; X)} \leq 2^{-100 n}\left\|\sum_{P \in \mathrm{T}^{-, n}} \widetilde{S_{P_{k}}} f\right\|_{L^{q}(\mathbb{R} ; \mathcal{X})} \leq 2^{-100 n}\left|I^{n}\right|^{\frac{1}{q}} \operatorname{eng}_{k}(f)(\mathrm{T} ; q) \leq 2^{-99 n}|I|^{\frac{1}{q}} \operatorname{eng}_{k}(f)(\mathrm{T} ; q)
$$

where the second bound holds because the operator inside the norm is a tree operator. Summation of the above bounds over $n$ yields the required control for the left hand side of (5.1). This estimate completes the proof of a. and b. parts of the Proposition. 
5.2. Proof of Proposition 4.1, a. and c. part: overlapping case. We keep the convention of writing $q$ for $q_{X_{k}}$ and $\mathcal{X}$ for $\mathcal{X}_{k}$.

Let $j \in \mathbf{j}_{\mathrm{T}}$. In this proof, we use the notation of [28, Section 4] for ${ }^{1}$ the sets $\tilde{E}_{j}$, the collections $\Omega_{j}$, the intervals $I_{j}^{\ell}, I_{j}^{r}$, to which we send for a detailed definition. There is no loss in generality with assuming that inf $\mathbf{j}_{\mathbf{T}}=0$, this corresponds to the normalization $\ell\left(I_{\top}\right)=1$. Define for $x \in \tilde{E}_{0}, j(x)=\max \left\{j \in \mathbf{j}_{\mathrm{T}}: x \in \tilde{E}_{j}\right\}$. The scale $2^{-j(x) J}$ is the smallest spatial scale relevant for $x$. It is logical to choose $2^{j(x) J}$ as the frequency scale for the cutoff at $x$, motivating the definition of

$$
\widetilde{\Pi_{k}} f:=\mathbf{1}_{\tilde{E}_{0}} T_{j(x)} f=\mathbf{1}_{\tilde{E}_{0}} T_{0} f+\sum_{j \geq 1} \mathbf{1}_{\tilde{E}_{j}} S_{j} f
$$

where the nestedness of $\tilde{E}_{j}$ and telescoping have been used to get the second equality. The construction of the actual phase-space projection operator $\Pi_{k}$ is made by suitably modifying $\widetilde{\Pi}_{k}$ and begins now.

Fix a scale $j \in \mathbf{j}_{\mathbf{T}}$ and a connected component $I=\left[x_{I}^{\ell}, x_{I}^{r}\right] \in \Omega_{j}$. The perturbation of $g_{j}=\mathbf{1}_{I} S_{j} f$ is made by adding and subtracting two auxiliary pieces at spatial scale $2^{-j J}$ which kill the mean value of $g_{j}$ : details follow.

Recall from [28, Lemma 4.12] that $I_{j}^{\ell}$ [resp. $I_{j}^{r}$ ] are intervals of length $2^{-2} \ell(I)$ whose right endpoint [resp. left endpoint] sits to the left of $x_{I}^{\ell}$ [resp. to the right of $x_{I}^{r}$ ] at a distance of $2^{-2} \ell(I)$. These intervals are well separated, see [28, Lemma 4.12] over $I \in \Omega_{j}, j \in$ $\mathbf{j}$ т. Introduce bump functions $\phi_{I, j}^{\ell}\left[\right.$ resp. $\phi_{I, j}^{r}$ ] adapted to $I_{j}^{\ell}\left[\operatorname{resp}\right.$. adapted to $\left.I_{j}^{r}\right]$ with normalization

$$
\int \phi_{I, j}^{\ell}=\int \phi_{I, j}^{r}=2^{-J j} .
$$

Decomposing $\mathbf{1}_{I}(x)=H_{I}^{\ell}(x)+H_{I}^{r}(x):=H\left(x-x_{I}^{\ell}\right)-H\left(x-x_{I}^{r}\right)$, where $H$ stands for Heaviside function. We introduce the $\mathcal{X}$-valued coefficients

$$
c_{I, j}^{\star}:=2^{I j} \int H_{I}^{\star} S_{j} f, \quad \star \in\{\ell, r\} .
$$

Before the next lemma, by combining [28, Lemmata 4.10,4.11], we realize that for $\star \in\{\ell, r\}$ there are tiles $P^{\star} \in \mathrm{T}$ with $\ell\left(I_{P^{\star}}\right) \sim 2^{-j J}$ and $\operatorname{dist}\left(I_{P^{\star}},\left\{x_{I}^{\star}\right\}\right) \lesssim 2^{-j J}$.

5.4. Lemma. Let $\chi \in X_{I_{p^{\star}}}, \star \in\{\ell, r\}$. We have the estimate

$$
\left|c_{I, j}^{\star}\right| \chi \lesssim \frac{1}{\left|I_{P^{\star}}\right|} \int\left|\chi(x) S_{j} f(x)\right|_{\mathcal{X}} \mathrm{d} x
$$

and in particular

$$
\left|c_{I, j}^{\star}\right| x \lesssim \operatorname{eng}_{k}(f)(\mathrm{T} ; q)
$$

\footnotetext{
${ }^{1}$ The collection $\mathbf{I}_{\mathrm{T}}$ is made of those J-dyadic intervals with the properties: 1) $3 I$ does not contain any $I_{P}$ with $P \in \mathrm{T}$; 2) the $J$-dyadic parent of $I$ fails 1$)$. Then $\tilde{E}_{j}=\cup\left\{I \in \mathbf{I}_{\mathrm{T}}: \ell(I)<2^{-j J}\right\}$. The set $\tilde{E}_{j}$ is a union of $J$-dyadic intervals of length $2^{-j J}$, and obviously $E_{j+1} \subset E_{j}$. The collection of the connected components of $E_{j}$ is referred to as $\Omega_{j}$.
} 
Proof. The first inequality is proved in the same fashion as [28, eq. (65)]. For the second, choose $\chi \in X_{I_{P^{\star}}}$ and note that $\chi^{2} \in X_{I_{P^{\star}}}$ as well. Hölder inequality yields

$$
\frac{1}{\left|I_{P^{\star}}\right|}\left|\chi^{2}(x) S_{j} f(x)\right|_{\chi} \mathrm{d} x \leq \frac{1}{\left|I_{P^{\star}}\right|}\|\chi\|_{q^{\prime}}\left\|\chi S_{j}\right\|_{L^{q}(\mathbb{R} ; \mathcal{X})} \sim \frac{1}{\left|I_{P^{\star}}\right|^{\frac{1}{q}}}\left\|\chi S_{j}\right\|_{L^{q}(\mathbb{R} ; \mathcal{X})} \lesssim \text { eng }_{k}(f)(\mathrm{T} ; q),
$$

where the last bound follows because $\chi S_{j} \in \mathbb{S}_{P^{\star}}$. This completes the proof.

With Lemma 5.1 in hand, we are able to define the phase-space projection operator: with reference to (5.2),

$$
\Pi_{k} f:=\widetilde{\Pi}_{k} f-\sum_{j \in \mathbf{j}} \sum_{I \in \Omega_{j}} \sum_{\star \in\{\ell, r\}} c_{I, j}^{\star} \phi_{I, j}^{\star}
$$

5.2.1. Proof of Proposition 4.1, part a. for $k \in B$. It suffices by interpolation to prove estimate (4.1) for $p=q$ together with the endpoint

$$
\left\|\Pi_{k} f\right\|_{L^{\infty}(\mathbb{R} ; X)} \lesssim \operatorname{eng}_{k}(f)(\mathrm{T} ; q) .
$$

Proof of (5.2.1). First of all, by virtue of the separation properties of the support of the $\phi_{I, j}^{\star}$ over $I \in \Omega_{j}, j \in \mathbf{j}_{\top}$ we have recalled earlier, and of the second bound in Lemma 5.1,

$$
\left\|\sum_{j \in \mathbf{j} \boldsymbol{T}} \sum_{I \in \Omega_{j}} \sum_{\star \in\{\ell, r\}} c_{I, j}^{\star} \phi_{I, j}^{\star}\right\|_{L^{\infty}(\mathbb{R} ; X)} \lesssim \operatorname{eng}_{k}(f)(\mathrm{T} ; q) .
$$

Hence, it suffices to prove an $L^{\infty}$ bound on $\widetilde{\Pi}_{k}$. Fix $x \in \tilde{E}_{0}$ and set $j=j(x)$. By construction of $j(x)$ there is an interval $I^{\prime} \subset \tilde{E}_{j}$ of length $2^{-j J}$ containing $x$, and by construction of $\mathbf{I}_{\top}$ there is a tile $P \in \mathrm{T}$ with $\ell\left(I_{P}\right) \sim 2^{-j J}$ and $I_{P} \subset 10 I^{\prime}$. Picking $\chi_{P} \in X_{I_{P}}$, writing $\alpha=\frac{1}{q}$ as before and using Lemma 2.2 in the second inequality

$$
\left|\widetilde{\Pi_{k}} f(x)\right|_{\mathcal{X}} \lesssim \chi_{P}\left|T_{j} f(x)\right|_{X} \lesssim\left|I_{P}\right|^{-\alpha}\left\|\chi_{P} T_{j} f\right\|_{L^{q}(\mathbb{R} ; \chi)} \lesssim \operatorname{eng}_{k}(f)(\mathrm{T} ; q) .
$$

Here the last bound comes from the fact that $g \mapsto \chi_{P} T_{j} g \in \mathbb{S}_{P}$ and $\{P\} \subset \mathrm{T}$ is a lacunary tree. This completes the proof of (5.2.1).

Proof of (4.1) for $p=q$. First of all, using the disjointness of $I \in \Omega_{j}, j \in \mathbf{j}_{\top}$ we estimate the $L^{q}(\mathbb{R} ; \mathcal{X})$-norm of the part involving the $\phi_{I, j}^{\star}$ by

$$
\left(\sum_{j \in \mathbf{j} \boldsymbol{\top}} \# \Omega_{j} 2^{-j J}\right)^{\frac{1}{q}}\left(\sup _{\star \in\{\ell, r\}} \sup _{\substack{j \in \mathbf{j} \mathbf{T} \\ I \in \Omega_{j}}}\left|c_{I, j}^{\star}\right| \chi\right) \lesssim\left|I_{T}\right|^{\frac{1}{q}} \text { eng }_{k}(f)(\mathrm{T} ; q) .
$$

where the first factor is bounded directly by [28, Lemma 4.12] while the second is (5.1) from Lemma 5.1. We are then left with proving

$$
\left\|\widetilde{\Pi}_{k} f\right\|_{L^{q}(\mathbb{R} ; X)}^{q} \lesssim\left|I_{\top}\right| \operatorname{eng}_{k}(T)(f ; q)^{q} .
$$

To prove (5.2.1) we recall that the sets $\tilde{E}_{j}$ are decreasing in $j$ and each is a union of disjoint intervals $I \in \mathbf{I}_{j}$ with $\ell(I)=2^{-j J}\left[28\right.$, Lemma 4.10]. Thus, the sets $E_{I}=I \cap\left(\tilde{E}_{j} \backslash \tilde{E}_{j+1}\right), I \in \mathbf{I}$ are 
a disjoint cover of each $E_{j} \backslash E_{j+1}$, and the latter sets are also pairwise disjoint and cover the support of $\widetilde{\Pi}_{k} f$. Furthermore,

$$
\sum_{j \in \mathbf{j}_{\top}} \sum_{I \in \mathbf{I}_{j}}|I| \lesssim\left|I_{\top}\right|
$$

as $\left|E_{I}\right| \geq 2^{-J}|I|$. In modern terms, the collection $\bigcup_{j} \mathbf{I}_{j}$ is $2^{-J}$ sparse. Indeed, for each $I \in \mathbf{I}_{j}$ we may find $I^{\prime} \subset \tilde{E}_{j} \backslash \tilde{E}_{j+1}$ with $I^{\prime} \subset I$ and $\ell\left(I^{\prime}\right)=2^{-J} \ell(I)$; see [28, Lemma 4.10]. As $\widetilde{\Pi}_{k} f(x)=T_{j} f$ for $x \in \widetilde{E}_{j} \backslash \tilde{E}_{j+1}$, the left hand side of (5.2.1) is controlled by

$$
\sum_{j \in \mathbf{j}_{\mathbf{T}}} \sum_{I \in \mathbf{I}_{j}}\left\|\mathbf{1}_{E_{I}} T_{j} f\right\|_{L^{q}(\mathbb{R} ; X)}^{q} \lesssim \sum_{j \in \mathbf{j}_{\mathbf{T}}} \sum_{I \in \mathbf{I}_{j}}\left\|\chi_{I} T_{j} f\right\|_{L^{q}(\mathbb{R} ; X)^{\prime}}^{q} \quad \chi_{I} \in X_{I} .
$$

By virtue of the last display and of (5.2.1), it suffices to show that

$$
\left\|\chi_{I} T_{j} f\right\|_{L^{q}(\mathbb{R} ; X)}^{q} \lesssim|I| \operatorname{eng}_{k}(f)(\mathbf{T} ; q)^{q}, \quad \forall j \in \mathbf{j}_{\top}, I \in \mathbf{I}_{j} .
$$

Fix such $j, I$. We now appeal to [28, Lemma 4.11] to find $P \in \mathrm{T}$ with $I_{P} \subset 10 I$ and $\ell\left(I_{P}\right) \lesssim 2^{-j J}$. As $\chi_{I} \in \widetilde{X_{I_{P}}}, f \mapsto \chi_{I} T_{j} f$ belongs to $\mathbb{S}_{P}$ and thus is a tree operator for $\{P\} \subset \mathrm{T}$, and the last display follows, completing the proof of (4.1).

5.2.2. Proof of Proposition 4.1, part c. We begin the proof by using the single scale estimate of Lemma 4.2. In fact (4.1) follows immediately from (4.1), (4.2) and the fact that $\mu_{j}$ is uniformly bounded. So it remains to prove (4.1). As usual, we prove the extremal cases. In fact, it suffices to prove the case $p=q$, as the case $p=\infty$ may then be recovered from Lemma 2.2.

Proof of (4.1) for $p=q$. In the proofs that follow, we use the local notation

$$
S_{P_{k}} g=\chi_{P} T_{m_{P_{k}}} g, \quad O g=\sum_{P \in \mathrm{T}\left(j_{0}\right)} S_{P_{k} g},
$$

where $\chi_{P} \in X_{I_{P}}=X_{I_{P}}(2 N, \delta, C, c)$ and $m_{P_{k}} \in M_{\omega_{P_{k}}}$. Notice that $O$ is a tree operator and as thus is bounded on $L^{q}\left(\mathbb{R} ; \mathcal{X}_{k}\right)$, but it is also pointwise bounded by maximal averages and thus bounded on $L^{\infty}\left(\mathbb{R} ; \mathcal{X}_{k}\right)$.

The first step in the proof proper is to notice that

$$
O\left(f-\Pi_{k} f\right)=O\left(T_{j_{0}} f-\Pi_{k} f\right),
$$

leading to the key is the decomposition

$$
\begin{aligned}
T_{j_{0}} f-\Pi_{k} f & = \\
& \mathbf{1}_{\mathbb{R} \backslash \tilde{E}_{j_{0}}} T_{j_{0}} f \\
& -\mathbf{1}_{\mathbb{R} \backslash \tilde{E}_{j_{0}}} \widetilde{\Pi}_{k} f \\
& +\sum_{\star \in\{\ell, r\}} \mathbf{1}_{\mathbb{R} \backslash \tilde{E}_{j_{0}}} \sum_{j \leq j_{0}} \sum_{I \in \Omega_{j}} c_{I, j}^{\star} \phi_{I, j}^{\star} \\
& -\sum_{\star \in\{\ell, r\}} \sum_{j>j_{0}} \sum_{I \in \Omega_{j}}\left(H_{I}^{\star} S_{j} f-c_{I, j}^{\star} \phi_{I, j}^{\star}\right) .
\end{aligned}
$$


cf. [28, eqs. (77)-(82)]. We now have to estimate the four contributions separately, and, as in [28], distinguish the local case $5 I_{0} \cap \tilde{E}_{j_{0}} \neq \emptyset$ from the complementary nonlocal case: for clarity, we first present the local case, and at the end of the proof we elaborate on the sketch provided in [28, p. 295] and unify the two cases: see Remark 5.2 below.

We first estimate the contribution of $g=(5.2 .2)-(5.2 .2)+(5.2 .2)$. Using the decay at scale $\ell\left(I_{0}\right)$ of the kernel of $O$ together with the $L^{\infty}$ bounds (5.2.1), (5.2.1), (5.1)

$$
\left\|\mathbf{1}_{I_{0}} O\left(\mathbf{1}_{\mathbb{R} \backslash 3 I_{0}} g\right)\right\|_{L^{q}\left(\mathbb{R} ; X_{k}\right)} \lesssim\left|I_{0}\right|^{\frac{1}{q}} \sum_{j \leq j_{0}} 2^{-J\left|j-j_{0}\right|}\left\langle\frac{\operatorname{dist}\left(I_{0}, \partial \tilde{E}_{j}\right)}{\ell\left(I_{0}\right)}\right\rangle^{-100} \text { eng }_{k}(f)(\mathrm{T} ; q)
$$

which is acceptable for (4.1). Further, if $\mathbf{1}_{3 I_{0}} g$ is nonzero, then $I_{0}$ is close to the boundary of $\tilde{E}_{j_{0}}$. In this case the right hand side of (4.1) is $O(1)$ and we may just aim for the estimate

$$
\left\|O\left(\mathbf{1}_{3 I_{0}} g\right)\right\|_{L^{q}\left(\mathbb{R} ; X_{k}\right)} \lesssim\left|I_{0}\right|^{\frac{1}{q}}\|O\|_{L^{q}\left(\mathbb{R} ; X_{k}\right)} \text { eng }_{k}(f)(\mathrm{T} ; q) .
$$

Although the $O$-norm appearing here is $O(1)$, we choose to keep this constant in evidence for later use.

We begin the proof of (5.2.2). We argue separately for each summand of $g$. First of all, we bound the contribution of (5.2.2). Appealing to [28, Lemma 4.11], we learn that there exists $P \in \mathrm{T}\left(j_{0}\right)$ such that $\operatorname{dist}\left(I_{0}, I_{P}\right) \sim 1$, so that for suitable choice of $\chi_{I_{0}} \in X_{I_{0}}$,

$$
\left\|\mathbf{1}_{3 I_{0}} T_{j_{0}} f\right\|_{L^{q}\left(\mathbb{R} ; X_{k}\right)} \leq\left\|\chi_{I_{0}} T_{j_{0}} f\right\|_{L^{q}\left(\mathbb{R} ; X_{k}\right)} \lesssim\left|I_{0}\right|^{\frac{1}{q}} \text { eng }_{k}(f)(\mathrm{T} ; q) .
$$

This makes the contribution of (5.2.2) acceptable for (5.2.2). To control the contribution of (5.2.2) we note that $\left(\mathbb{R} \backslash \tilde{E}_{j_{0}}\right) \cap 3 I_{0}$ is the union of at most three intervals $I_{1}$ of length $\ell\left(I_{0}\right)$, on which $\widetilde{\Pi_{k}} f$ coincides with $T_{j_{0}-1} f$. On each of these intervals, by the same argument used for (5.2.2),

$$
\left\|\mathbf{1}_{I_{1}} T_{j_{0}-1} f\right\|_{L^{q}\left(\mathbb{R} ; X_{k}\right)} \lesssim\left|I_{0}\right|^{\frac{1}{q}} \text { eng }_{k}(f)(\mathrm{T} ; q)
$$

which is acceptable. Finally, from the last claim of [28, Lemma 4.12] we gather that $I_{j}^{\star} \cap 3_{I_{0}} \neq \varnothing$ for at most $O(1)$ intervals $I \in \Omega_{j}$ with $j \leq j_{0}$. Therefore

$$
\left\|\mathbf{1}_{3 I_{0}}(5.2 .2)\right\|_{L q\left(\mathbb{R} ; X_{k}\right)} \lesssim\left|I_{0}\right|^{\frac{1}{q}} \sup _{I, j, \star}\left|c_{I, j}\right| \lesssim\left|I_{0}\right|^{\frac{1}{q}} \operatorname{eng}_{k}(f)(\mathrm{T} ; q)
$$

by (5.1), and we have proved (5.2.2). This finishes the control of terms (5.2.2) to (5.2.2).

To complete the proof of (4.1), we are left with estimating the small spatial scales term (5.2.2). Using the triangle inequality and the definition of $\mu_{j_{0}}$, it will suffice to prove that for each fixed $\star \in\{\ell, r\}, j>j_{0}, I \in \Omega_{j}$ there holds

$$
\begin{aligned}
& \left\|\mathbf{1}_{I_{0}} O\left(G_{I}\right)\right\|_{L^{q}\left(\mathbb{R} ; \chi_{k}\right)} \lesssim\left|I_{0}\right|^{\frac{1}{q}} \mathrm{eng}_{k}(f)(\mathrm{T} ; q) \int \frac{\chi_{I_{0}}(x)}{\left|I_{0}\right|} 2^{-\frac{\left(j-j_{0}\right)}{100}}\left\langle 2^{j J}\left|x-x_{I}^{\star}\right|\right\rangle^{-100} \mathrm{~d} x, \\
& G_{I}:=H_{I}^{\star} S_{j} f-c_{I, j}^{\star} \phi_{I, j}^{\star} .
\end{aligned}
$$

As they will be kept fixed below, we have omitted $\star$ and $j$ from the $G_{I}$ notation for simplicity. Let $n \in \mathbb{N}$ be the least integer such that $2^{n} I_{0} \cap I_{j}^{\star} \neq \varnothing$. A direct computation of the right hand side and the fact that $\chi_{I_{0}} \in X_{I_{0}}$ tells us that the above bound is equivalent to the estimate

$$
\left\|\mathbf{1}_{I_{0}} O\left(G_{I}\right)\right\|_{L^{q}\left(\mathbb{R} ; X_{k}\right)} \lesssim\left|I_{0}\right|^{\frac{1}{q}} 2^{-\frac{\left(j-j_{0}\right)}{100}} 2^{-j J} 2^{-100 n}
$$


The final stretch of the proof will be to establish (5.2.2). As the frequency support of $O$ is localized near $2^{j_{0}}$, we gather that $O\left[\left(T_{j-1} H_{I}^{\star}\right)\left(S_{j} f\right)\right]=0$. This means we may replace $G_{I}$ by

$$
F_{I}=G_{I}-\left(T_{j-1} H_{I}^{\star}\right)\left(S_{j} f\right)=\left[\left(1-T_{j-1}\right) H_{I}^{\star}\right] S_{j} f-c_{I, j}^{\star} \phi_{I, j}^{\star} .
$$

As both $G_{I}$ and $F_{I}-G_{I}$ have mean zero, $F_{I}$ also does. Letting $\Phi_{I}$ be the antiderivative of $F_{I}$ which vanishes at $\pm \infty$, we have

$$
O F_{I}(x)=2^{J j_{0}} \widetilde{O} \Phi(x),
$$

where $\widetilde{O}$ is the pseudodifferential operator

$$
\widetilde{O} g(x)=\sum_{P \in \mathrm{T}\left(j_{0}\right)} \int \chi_{I_{P}}(x) 2^{-J j_{0}} \xi m_{P_{k}}(\xi) \widehat{g}(\xi) \mathrm{e}^{i x \xi} \mathrm{d} \xi
$$

In fact $\widetilde{O}$ is a tree operator as $\xi \mapsto 2^{-J j_{0}} \xi m_{P_{k}}(\xi)$ belongs to $M_{\omega_{P_{k}}}$. Therefore, we begin to bound (4.1) by

$$
\left.\| \mathbf{1}_{I_{0}} O G_{I}\right)\left\|_{L q\left(\mathbb{R} ; \mathcal{X}_{k}\right)} \lesssim\right\| \chi_{I_{0}} \Phi_{I} \|_{L q\left(\mathbb{R} ; \mathcal{X}_{k}\right)} .
$$

An estimate on $\left|\Phi_{I}(x)\right|_{X}$ compatible with the right hand side of (5.2.2) may be produced, cf. [28, p. 298], once we establish the pointwise bound

$$
\left|F_{I}(x)\right|_{X} \lesssim \operatorname{eng}_{k}(f)(\mathrm{T} ; q)\left\langle 2^{j J}\left|x-x_{I}^{\star}\right|\right\rangle^{-100} .
$$

The last step towards (5.2.2), and therefore (4.1), is to prove (5.2.2). The contribution of $c_{I, j}^{\star} \phi_{I, j}^{\star}$ is controlled by virtue of the decay of $\phi_{I, j}^{\star}$ and (5.1). We turn to controlling the summand $\left[\left(1-T_{j-1}\right) H_{I}^{\star}\right] S_{j} f$. First we recall that by construction of $I \in \Omega_{j}$ and $I_{j}^{\star}$, we may find a dyadic interval $I^{\prime}$ with $\ell\left(I^{\prime}\right)=2^{-j J}$ adjacent to the left endpoint of $I$, in particular $\operatorname{dist}\left(x_{I}^{\ell}, I^{\prime}\right) \sim 2^{-j}$, and $P \in \mathrm{T}$ such that $I_{P} \subset I^{\prime}$. Pick $\chi^{\prime} \in X_{I}$ with decay parameter $N$ (for instance). As $g \mapsto \chi_{I^{\prime}} S_{j} g \in \mathbb{S}_{P}$ and $\{P\} \subset \mathrm{T}$, we have the estimate

$$
\left\|\chi_{I^{\prime}} S_{j} f\right\|_{L^{\infty}(\mathbb{R} ; X)} \leq \frac{1}{\left|I^{\prime}\right|^{\frac{1}{q}}}\left\|\chi_{I^{\prime}} S_{j} f\right\|_{L^{q}(\mathbb{R} ; X)} \lesssim \operatorname{eng}_{k}(f)(\mathrm{T} ; q)
$$

where the first inequality is Lemma 2.2. As $\operatorname{dist}\left(x_{I^{\ell}}^{\ell} I^{\prime}\right) \sim 2^{-j}$, we have that $\chi_{I^{\prime}}(x) \gtrsim$ $\left\langle 2^{j J}\left|x-x_{I_{j}^{\star}}\right|\right\rangle^{-N}$. Putting these estimates together,

$$
\left|S_{j} f(x)\right| \lesssim\left\langle 2^{j J}\left|x-x_{I_{j}^{\star}}\right|\right\rangle^{N} \text { eng }_{k}(f)(T ; q) .
$$

Integrating repeatedly by parts the high frequency function $\left[\left(1-T_{j-1}\right) H_{I}^{\star}\right]$, we may bound it pointwise by factors of $\lesssim_{N}\left\langle 2^{j J}\left|x-x_{I}^{\star}\right|\right\rangle^{-N-100}$, compensating the polynomial growth of the last display and yielding an acceptable right hand side for (5.2.2) which is finally proved. The proof of (4.1) is finally complete.

5.20. Remark (The nonlocal case of (4.1)). The local/nonlocal cases can be unified by introduction of the parameter

$$
Z=\text { least nonnegative integer such that } I_{0} \pm Z \ell\left(I_{0}\right) \cap \tilde{E}_{j_{0}} \neq \emptyset .
$$


Comparing with what we did to obtain (5.2.2), and to [28, Lemma 4.11], we learn that there exists $P \in \mathrm{T}\left(j_{0}\right)$ such that $\operatorname{dist}\left(I_{0}, I_{P}\right) \sim Z$, so that

$$
\text { eng }_{k}(f)\left(\mathbb{P}\left(I_{0}\right) ; q\right) \lesssim Z^{N}, \quad \mathbb{P}\left(I_{0}\right)=\left\{P \text { is any tri-tile with } I_{P}=I_{0}\right\} .
$$

This introduces a $Z^{N}$ loss in e. g. estimates (5.2.2), (5.2.2). However, as we are concerned with estimates for $1_{I_{0}} O\left(T_{j_{0}} f-\Pi_{k} f\right)$, we may replace $O$ by the operator $g \mapsto \tilde{O} g=\chi_{I_{0}} O g$, where $\chi_{I_{0}} \in X_{I_{0}}(2 N, \delta, C, c)$ and $\chi_{I_{0}} \geq \mathbf{1}_{I_{0}}$. The separation between $\tilde{E}_{j_{0}}$ and $I_{0}$ yields that

$$
\|\tilde{O}\|_{L q\left(\mathbb{R} ; X_{k}\right)} \lesssim Z^{-2 N},
$$

and the same additional decay factor is gained in the kernel estimates for $\tilde{O}$. Replacing $O$ by $\tilde{O}$ in (5.2.2), (5.2.2) and taking (5.2) into account offsets the loss introduced by (5.2).

\section{Proof of Lemma 3.2}

We begin with a definition. We say that a family of trees $T \in T$ is $k$-strongly disjoint with parameter $\beta \geq 1$ if

i. each $T$ is a $k$-lacunary tree;

ii. if $\mathrm{T}, \mathrm{T}^{\prime} \in \mathrm{T}, \mathrm{T} \neq \mathrm{T}^{\prime}$, then

$$
P \in \mathrm{T}, P^{\prime} \in \mathrm{T}^{\prime}, \ell\left(\omega_{P}\right) \leq \ell\left(\omega_{P^{\prime}}\right), 10 \beta \omega_{P_{k}} \cap 10 \beta \omega_{P_{k}^{\prime}} \neq \varnothing \Longrightarrow I_{P^{\prime}} \cap I_{\top}=\varnothing .
$$

The rationale behind this definition is that, if the consequence of the above implication failed, the tri-tile $P^{\prime}$ would qualify to be in a suitable completion of the tree $T$. In what follows, we work with the parameter $\beta=1$, as the general case $1 \leq \beta \ll 2^{J}$ may be handled by finite splitting.

6.1. The $L^{2}$-orthogonality estimates. Tree operators associated to families of $k$-strongly disjoint trees give rise to an $L^{2}$ almost orthogonality estimate: this is well known, and extends to the case of Hilbert space valued functions, as detailed in the next lemma. This lemma is a transposition of [19, Proposition 6.1] to our context. It is convenient in what follows to introduce the single tile version of the energy parameters. To do so, for each tri-tile $P$ we introduce the function

$$
u_{P}:=\left\langle\frac{\left|x-c\left(I_{P}\right)\right|}{\ell\left(I_{P}\right)}\right\rangle^{-10}
$$

and define

$$
\begin{aligned}
& \|f\|_{P, k, q}=\sup _{m_{P_{k}} \in M_{P_{k}}}\left|I_{\mathrm{P}}\right|^{-\frac{1}{q}}\left\|u_{P} T_{m_{P_{k}}} f\right\|_{L^{q}(\mathbb{R} ; \mathcal{X})^{\prime}}, \\
& \text { eng }_{k, s s}(f)(\mathbb{P} ; q):=\sup _{P \in \mathbb{P}}\|f\|_{P, k, q} .
\end{aligned}
$$

For uniformity, we gave the definitions above for a generic $1 \leq q \leq \infty$. However, Lemma 2.2 shows the upper bound $\|f\|_{P, k, \infty} \lesssim\|f\|_{P, k, 1}$, and it follows that $\|f\|_{P, k, p} \sim_{p, q}\|f\|_{P, k, q}$ for all $1 \leq p, q \leq \infty$. Below, we will only use the value $q=2$ in (6.1). Note the trivial bounds

$$
\begin{aligned}
& \|f\|_{P, k, q} \lesssim\left|I_{P}\right|^{-\frac{1}{q}}\left\|u_{P}\right\|_{q} \sup _{m_{P_{k}} \in M_{P_{k}}}\left\|T_{m_{P_{k}}} f\right\|_{L^{\infty}(\mathbb{R} ; \mathcal{X})} \lesssim\|f\|_{L^{\infty}(\mathbb{R} ; \mathcal{X}),} \\
& \sup _{S_{P_{k}} \in \mathbb{S}_{P_{k}}}\left\|S_{P_{k}} f\right\|_{L^{q}(\mathbb{R} ; \mathcal{X})} \lesssim\left|I_{P}\right|^{\frac{1}{q}}\|f\|_{P, k, q} .
\end{aligned}
$$


6.5. Lemma. Let $X$ be a Hilbert space and $\mathbf{T}$ be a collection of $k$-strongly disjoint trees, and define $\mathbb{T}=\bigcup\{T: T \in T\}$. There holds

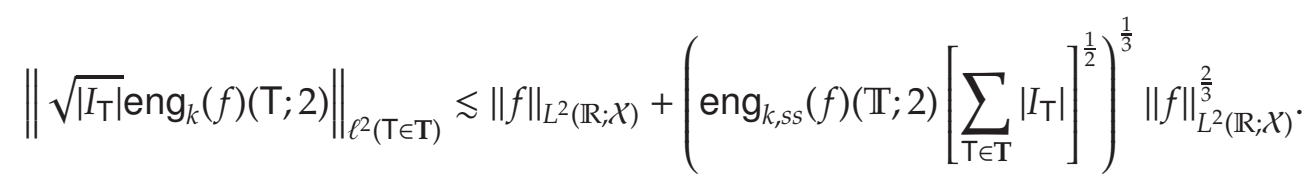

Before the proof proper, we enucleate the almost orthogonality of the single tile operators within a $k$-lacunary tree.

6.7. Lemma. Let $\mathcal{X}$ be a Hilbert space and $\mathrm{T}$ be a k-lacunary tree. Then

$$
\sqrt{\left|I_{\top}\right|} \operatorname{eng}_{k}(f)(\mathrm{T} ; 2) \lesssim \sqrt{\sum_{P \in \mathrm{T}}\left|I_{P}\right|\|f\|_{P, k, 2}^{2}}
$$

Proof. By modulation invariance, it suffices to take care of the case $\xi_{T}=0$. Choose a tree operator $T_{\mathrm{T}}=\sum_{P \in \mathrm{T}} S_{P_{k}}$ that nearly achieves the supremum in eng $\mathrm{e}_{k}(f)(\mathrm{T} ; 2)$ and write $S_{P_{k}} g=\chi_{P} T_{m_{P_{k}}} g$. From the disjointness of the frequency supports, we have that, referring to $(3.1)$

$$
\left\langle S_{P_{k}} f, S_{P_{k}^{\prime}} f\right\rangle \neq 0 \Longrightarrow P, P^{\prime} \in \mathrm{T}(j)
$$

For $n \in \mathbb{Z}$ denote by $P^{+n}$ the (at most) unique tri-tile $P^{\prime} \in \mathrm{T}(j)$ with $I_{P^{\prime}}=I_{P}+n \ell\left(I_{P}\right)$. Then define

$$
\tilde{\chi}_{P}:=\frac{\chi_{P}}{u_{P}}, \quad \tilde{S}_{P_{k}} g:=\tilde{\chi}_{P} T_{m_{P_{k}}} g .
$$

It is immediate to see that $\tilde{\chi}_{P} \in \Psi_{I_{P}}$ as multiplying by a polynomial does not change the frequency support neither significantly alters the rapid decay of $\chi_{P}$, hence $\tilde{S}_{P_{k}}$ belongs to $\mathbb{S}_{P_{k}}$. Therefore

$$
\begin{aligned}
\left|I_{\mathbf{T}}\right| \text { eng }_{k}(f)(\mathrm{T} ; 2)^{2} & \lesssim\left\|T_{\mathrm{T}} f\right\|_{L^{2}(\mathbb{R} ; \mathcal{X})}^{2} \lesssim \sum_{j \in \mathbf{j} \mathbf{T}} \sum_{P \in \mathrm{T}(j)} \sum_{n \in \mathbb{Z}} \int S_{P_{k}} f \overline{S_{P_{k}^{+n} f} f} \\
& \leq \sum_{j \in \mathbf{j} \boldsymbol{\top}} \sum_{P \in \mathrm{T}(j)} \sum_{n \in \mathbb{Z}} \int\left|\tilde{S}_{P_{k}} f \| \tilde{S}_{P_{k}^{+n}} f\right| u_{P} u_{P^{+n}} \\
& \lesssim \sum_{j \in \mathbf{j} \boldsymbol{T}} \sum_{P \in \mathrm{T}(j)} \sum_{n \in \mathbb{Z}}\langle n\rangle^{-10}\left(\left\|\tilde{S}_{P_{k}} f\right\|_{L^{2}(\mathbb{R} ; \mathcal{X})}^{2}+\left\|\tilde{S}_{P_{k}^{+n}} f\right\|_{L^{2}(\mathbb{R} ; \mathcal{X})}^{2}\right) \\
& \lesssim \sum_{P \in \mathrm{T}}\left\|\tilde{S}_{P_{k}} f\right\|_{L^{2}(\mathbb{R} ; \mathcal{X})}^{2} \lesssim \sum_{P \in \mathrm{T}}\left|I_{P}\right|\|f\|_{P, k, 2}^{2}
\end{aligned}
$$

and this proves the claimed inequality. We have used $\left\|u_{P} u_{P+n}\right\|_{\infty} \lesssim\langle n\rangle^{-10}$ to pass to the second line and (6.1) in the last estimate.

Proof of Lemma 6.1. Let us choose the scaling $\|f\|_{L^{2}(\mathbb{R} ; X)}=1$. From Lemma 6.2, we may bound the quantity

$$
S:=\sqrt{\sum_{P \in \mathbb{T}}\left|I_{P}\right|\|f\|_{P, k, 2}^{2}}
$$


in place of the left hand side of (6.1). Then

$$
S^{2} \sim \sum_{P \in \mathbb{T}}\left|I_{P}\right|\|f\|_{P, k, 2} \frac{\left\|u_{P} T_{m_{P_{k}}} f\right\|_{L^{2}(\mathbb{R} ; \mathcal{X})}}{\left|I_{P}\right|^{\frac{1}{2}}}
$$

having linearized the suprema in $\|f\|_{P, k, 2}$ with a suitable choice $m_{P_{k}} \in M_{P_{k}}, P \in \mathbb{T}$. From now on, as $m_{P_{k}}$ and $k$ are fixed, we simply write $T_{P}$ in place of $T_{m_{P_{k}}}$, and by using Lemma 2.2 , identifying $X^{\prime}$ with $X$ via Riesz representation, we have

$$
\frac{\left\|u_{P} T_{P} f\right\|_{L^{2}(\mathbb{R} ; X)}}{\left|I_{P}\right|^{\frac{1}{2}}}=\frac{1}{\left|I_{P}\right|}\left\langle T_{P}^{*} v_{P}, f\right\rangle, \quad P \in \mathbb{T}
$$

for some function $v_{P}$ with

$$
\left|v_{P}(x)\right|_{x} \lesssim u_{P}(x), \quad x \in \mathbb{R} .
$$

These considerations lead to the estimate

$$
S^{2} \sim\left\langle\sum_{P \in \mathbb{T}}\|f\|_{P, k, 2} T_{P}^{*} v_{P}, f\right\rangle \leq\left\|\sum_{P \in \mathbb{T}}\right\| f\left\|_{P, k, 2} T_{P}^{*} v_{P}\right\|_{L^{2}(\mathbb{R} ; \mathcal{X})} .
$$

Define now

$$
\mathbb{T}_{<}(P):=\left\{P^{\prime} \in \mathbb{T}: \omega_{P_{k}} \subsetneq \omega_{P_{k}^{\prime}}\right\}, \quad \mathbb{T}_{=}(P):=\left\{P^{\prime} \in \mathbb{T}: \omega_{P_{k}^{\prime}}=\omega_{P_{k}}\right\} .
$$

Frequency support considerations applied to the inner products $\left\langle T_{P}^{*} v_{P}, T_{P^{\prime}}^{*} v_{P^{\prime}}\right\rangle$ then lead to the chain of inequalities

$$
\begin{aligned}
& \left\|\sum_{P \in \mathbb{T}}\right\| f\left\|_{P, k, 2} T_{P}^{*} v_{P}\right\|_{L^{2}(\mathbb{R} ; \mathcal{X})}^{2} \\
= & \sum_{P \in \mathbb{T}} \sum_{P^{\prime} \in \mathbb{T}_{=}(P)}\|f\|_{P, k, 2}\|f\|_{P^{\prime}, k, 2}\left\langle T_{P}^{*} v_{P}, T_{P^{\prime}}^{*}, v_{P^{\prime}}\right\rangle \\
+ & 2 \sum_{P \in \mathbb{T}} \sum_{P^{\prime} \in \mathbb{T}_{<}(P)}\|f\|_{P, k, 2}\|f\|_{P^{\prime}, k, 2}\left\langle T_{P}^{*} v_{P}, T_{P^{\prime}}^{*}, v_{P^{\prime}}\right\rangle:=S_{1}+2 S_{2} .
\end{aligned}
$$

We first treat $S_{1}$. Note that if $P^{\prime} \in \mathbb{P}_{=}(P)$ then $P^{\prime}=P^{+n}$ for some $n \in \mathbb{Z}$, see the line before (6.1) for a definition. The decay of $v_{P}(6.1)$ and the kernel estimate for $T_{P}^{*}$ guarantee the pointwise bound

$$
\left|T_{P}^{*} v_{P}\right|_{x} \lesssim u_{P}
$$

whence

$$
\left|\left\langle T_{P}^{*} v_{P}, T_{P^{+n}}^{*} v_{P^{+n}}\right\rangle\right| \lesssim\left|I_{P}\right|\langle n\rangle^{-10}, \quad n \in \mathbb{Z} .
$$

Therefore, we control

$$
S_{1} \lesssim \sum_{P \in \mathbb{P}} \sum_{n \in \mathbb{Z}}\langle n\rangle^{-10}\left(\left|I_{P}\right|\|f\|_{P, k, 2}^{2}+\left|I_{P^{+n}}\right|\|f\|_{P^{+n}, k, 2}^{2}\right) \lesssim S^{2}
$$


using the definition of $S$. We turn to $S_{2}$. Notice that if $P^{\prime} \in \mathbb{T}_{<}(P)$, then $\ell\left(I_{P^{\prime}}\right)<\ell\left(I_{P}\right)$. Relying on (6.1) again

$$
\left|\left\langle T_{P}^{*} v_{P}, T_{P^{\prime}}^{*} v_{P^{\prime}}\right\rangle\right| \lesssim\left|I_{P^{\prime}}\right|\left\langle\frac{\operatorname{dist}\left(I_{P}, I_{P^{\prime}}\right)}{\ell\left(I_{P}\right)}\right\rangle^{-10} \lesssim\left\|\mathbf{1}_{I_{P^{\prime}}} u_{P}\right\|_{1}, \quad P^{\prime} \in \mathbb{T}_{<}(P) .
$$

Also note that, due to the condition ii. in the definition of the strongly disjoint trees, if $P^{\prime} \in \mathbb{T}_{<}(P)$ then $I_{P^{\prime}} \cap I_{T(P)}=\varnothing$ where $\mathrm{T}(P)$ is the unique tree in $\mathbb{T}$ where $P$ belongs, and furthermore if $P^{\prime}, P^{\prime \prime} \in \mathbb{T}_{<}(P)$ then $I_{P^{\prime}} \cap I_{P^{\prime \prime}}=\emptyset$ : this is a combination of conditions $i$. and ii. of the definition, see $[19,28]$ for details. Using definition (6.1), estimate (6.1), the trivial estimate $\left\|\mathbf{1}_{I_{P}} u_{P}\right\|_{1} \leq\left\|u_{P}\right\|_{1} \lesssim\left|I_{P}\right|$, Cauchy-Schwarz, disjointness and separation from $I_{T(P)}$ of $\left\{I_{P^{\prime}}: P^{\prime} \in \mathbb{T}_{<}(P)\right\}$, and we obtain

$$
\begin{aligned}
S_{2} & \lesssim \operatorname{eng}_{k, s s}(f)(\mathbb{T} ; 2) \sum_{P \in \mathbb{T}}\|f\|_{P, k, 2} \sum_{P^{\prime} \in \mathbb{T}_{<}(P)}\left\|\mathbf{1}_{I_{P^{\prime}}} u_{P}\right\|_{1} \\
& \leq \operatorname{eng}_{k, s s}(f)(\mathbb{T} ; 2) \sum_{P \in \mathbb{T}} \sqrt{\left|I_{P}\right|}\|f\|_{P, k, 2}\left\|\mathbf{1}_{\mathbb{R} \backslash I_{T}} u_{P}\right\|_{1}^{\frac{1}{2}} \\
& \lesssim \operatorname{eng}_{k, s s}(f)(\mathbb{T} ; 2)\left(\sum_{P \in \mathbb{T}} \mid I_{P}\|f\|_{P, k, 2}^{2}\right)^{\frac{1}{2}}\left(\sum_{\mathbb{T} \in \mathbf{T}} \sum_{P \in \mathbb{T}}\left\|\mathbf{1}_{\mathbb{R} \backslash I_{T}} u_{P}\right\|_{1}\right)^{\frac{1}{2}} \\
& \lesssim \operatorname{eng}_{k, s s}(f)(\mathbb{T} ; 2)\left(\sum_{\mathbb{T} \in \mathbf{T}}\left|I_{T}\right|\right)^{\frac{1}{2}} S ;
\end{aligned}
$$

we omitted some of the details, see e.g. [19, Proposition 6.1]. Summarizing (6.1), (6.1), (6.1), (6.1)

$$
S^{2} \lesssim \sqrt{S_{1}+2 S_{2}} \lesssim\left(S^{2}+\text { eng }_{k, s S}(f)(\mathbb{T} ; 2)\left(\sum_{\mathbf{T} \in \mathbf{T}}\left|I_{\mathrm{T}}\right|\right)^{\frac{1}{2}} S\right)^{\frac{1}{2}}
$$

which yields the claimed bound. The details can be read from [19, Proposition 6.1], hence we omit them.

6.2. Transporting almost orthogonality to interpolation spaces. In the previous subsection, we have shown that the definitions (3.3), (6.1) lead to Hilbert space valued orthogonality estimates for families of strongly disjoint trees. The point is that the definitions (3.3), (6.1) are of maximal nature and involve more general operators than the rank 1 projections $f \mapsto\left\langle f, \varphi_{P_{k}}\right\rangle \varphi_{P_{k}}$ of [19], namely operators of the class $\mathbb{S}_{P_{k}}$. It is because of this additional generality that we had to reproduce, with small changes, the classical $T T^{*}$ arguments of [19].

Now that our version of [19, Prop. 6.1], namely Lemma 6.1 is in place, the interpolation arguments of [19, Section 7] may be perused mutatis mutandis, leading to the following almost orthogonality estimate for interpolation spaces. 
6.16. Proposition. Let $2 \leq p<\infty$ and $\mathcal{X}=\left[\mathcal{Y}_{0}, \mathcal{Y}_{1}\right]_{\frac{2}{p}}$ be the complex interpolation space of a UMD space $\mathcal{Y}_{0}$ and a Hilbert space $\mathcal{Y}_{1}$. Then for all $0<\alpha \leq 1$ the inequality

$$
\left\|\left.|| I_{T}\right|^{\frac{1}{p}} \operatorname{eng}_{k}(f)(\mathrm{T} ; p)\right\|_{\ell^{p}(\mathrm{~T} \in \mathbf{T})} \lesssim_{\alpha}\|f\|_{L^{p}(\mathbb{R} ; \mathcal{X})}+\left(\|f\|_{L^{\infty}(\mathbb{R} ; X)}\left[\sum_{\mathbf{T} \in \mathbf{T}}\left|I_{\boldsymbol{T}}\right|\right]^{\frac{1}{p}}\right)^{1-\alpha}\|f\|_{L^{p}(\mathbb{R} ; X)}^{\alpha}
$$

holds uniformly over all collections $\mathbf{T}$ of $k$-strongly disjoint trees.

Proof. The first step of the proof consists of deducing the case $p=2$ of (6.3) from Lemma 6.1. With

$$
\text { eng }_{k, s s}(f)(\mathbb{T} ; 2) \lesssim\|f\|_{L^{\infty}(\mathbb{R} ; X)}
$$

in hand, a consequence of (6.1), this is accomplished following step by step the proof of [19, Proposition 6.6].

The second step consists in the deduction of an endpoint at $p=\infty$, which is

$$
\left\|\mathrm{eng}_{k}(f)(\mathrm{T} ; \mathrm{BMO})\right\|_{\ell^{\infty}(\mathrm{T} \in \mathbf{T})} \lesssim\|f\|_{L^{\infty}(\mathbb{R} ; \mathcal{X})}
$$

having denoted

$$
\|f\|_{k, \mathrm{~T}, \mathrm{BMO}}:=\sup \left\|\operatorname{Mod}_{-\xi_{\mathrm{T}}} T_{\mathrm{T}} f\right\|_{\mathrm{BMO}(\mathbb{R} ; \chi)^{\prime}} \quad \operatorname{eng}_{k}(f)(\mathrm{T} ; \mathrm{BMO}):=\sup _{\substack{\mathrm{T} \mathbb{} \\ \mathrm{T} k \text {-lacunary }}}\|f\|_{k, \mathrm{~T}, \mathrm{BMO}}
$$

where $\operatorname{Mod}_{-\xi}$ stands for modulation by $-\xi$, and usual the first supremum is taken over all possible choices of type $k$ tree operators $T_{\mathrm{T}}$. The estimate (6.2) is an immediate consequence of the uniform estimate for demodulated tree operators

$$
\operatorname{Mod}_{-\xi_{\mathrm{T}}} T_{\mathrm{T}} \operatorname{Mod}_{\xi_{\mathrm{T}}}: L^{\infty}(\mathbb{R} ; \mathcal{X}) \rightarrow \operatorname{BMO}(\mathbb{R} ; \mathcal{X}),
$$

which holds by virtue of the fact that each operator $\operatorname{Mod}_{-\xi_{T}} T_{T} \operatorname{Mod}_{\xi_{T}}$ is a CalderónZygmund operator.

Finally, the proof of the proposition is obtained by complex interpolation of the case $q=2$ of (6.3) with (6.2). Details are given in [19, Proposition 7.3].

6.3. The proof proper of Lemma 3.2. The proof proceeds via an iterative algorithm similar to [19, Proposition 8.4]. One additional remark necessary here is that the selected trees come from a greedy selection process, and therefore satisfy properties a. b. and c. of Subsection 3.1, cf. [28, Lemmata 4.4 and 4.7].

For the proof, write $p=q_{x}, \lambda:=\operatorname{eng}_{k}(f)(\mathbb{P} ; p)$ and let $\alpha \in(0,1)$ be chosen so that $q=p / \alpha$. Performing an iterative algorithm analogous to [27, Lemma 7.7], we decompose

$$
\mathbb{P}:=\mathbb{P}^{-} \cup \mathbb{P}^{+},
$$

where

$$
\operatorname{eng}_{k}(f)\left(\mathbb{P}^{-} ; p\right) \leq \frac{\lambda}{2}
$$

and $\mathbb{P}^{+}=\bigcup\{T: T \in T\}$ is a family of greedily selected trees with the following property: for each $T$ there exists a $k$-lacunary tree $T^{\prime} \subset T$ with $I_{T}=I_{T^{\prime}}$ and the family $\mathbf{T}^{\prime}=\left\{T^{\prime}: T \in \mathbf{T}\right\}$ consists of $k$-strongly disjoint trees with

$$
\operatorname{eng}_{k}(f)(\mathrm{T} ; p) \gtrsim \lambda
$$


Using Proposition 6.3 in the second inequality,

$$
\begin{aligned}
\lambda^{p} \sum_{\mathbf{T} \in \mathbf{T}}\left|I_{\mathbf{T}}\right| & \lesssim\left\|\left|I_{\mathbf{T}}\right|^{\frac{1}{p}} \operatorname{eng}_{k}(f)(\mathbf{T} ; p)\right\|_{\ell^{p}(\mathbf{T} \in \mathbf{T})}^{p} \lesssim\|f\|_{L^{p}(\mathbb{R} ; \mathcal{X})}^{p}+\|f\|_{L^{\infty}(\mathbb{R} ; \mathcal{X})}^{p(1-\alpha)}\left(\sum_{\mathbf{T} \in \mathbf{T}}\left|I_{\mathbf{T}}\right|\right)^{1-\alpha}\|f\|_{L^{p}(\mathbb{R} ; \mathcal{X})}^{\alpha p} \\
& \lesssim|F|+\left(\sum_{\mathbf{T} \in \mathbf{T}}\left|I_{T}\right|\right)^{1-\alpha}|F|^{\alpha}
\end{aligned}
$$

Dividing into cases depending on whether $|F|$ the summand in the last line is larger or not than the $|F|^{\alpha}$ one,

$$
\sum_{T \in \mathbf{T}}\left|I_{\top}\right| \lesssim \max \left\{\lambda^{-p}, \lambda^{-q}\right\}|F| \lesssim \lambda^{-q}|F|
$$

which is what we had to prove to conclude Lemma 3.2. In the last comparison we have used that $q>p$ and

$$
\lambda \lesssim \sup _{P \in \mathbb{P}} \inf _{I_{P}} \mathrm{M}\left(|f|_{X}\right) \lesssim 1,
$$

a consequence of Lemma 3.1. The proof of Lemma 3.2 is complete.

\section{Proof of Lemma 3.1}

Throughout this proof, if $I$ is a $J$-dyadic interval, we write $I^{+v}=I+v \ell(I)$ for $v \in \mathbb{Z}$ to denote the $v$-th translate of $I$. Further, we introduce the local notation

$$
\gamma_{I}(x):=\left\langle\frac{x-c(I)}{\ell(I)}\right\rangle^{100}, \quad x \in \mathbb{R} .
$$

The polynomial $\gamma_{I}$ will be used to apply the so-called localization trick. As we perform this a few times in the proof, we isolate the related notation here. If $\mathrm{T}$ is a $k$-lacunary tree and $T_{\mathrm{T}}$ a tree operator, we write

$$
\tilde{T}_{\mathrm{T}} g:=\sum_{P \in \mathrm{T}} \tilde{S}_{P_{k} g}, \quad \tilde{S}_{P_{k} g}:=\gamma_{I_{\mathrm{T}}} S_{P_{k}} g .
$$

It is immediate to verify that $\tilde{S}_{P_{k}} \in \mathbb{S}_{P_{k}}$ for all $P \in \mathrm{T}$, so that $\tilde{T}_{\mathrm{T}}$ is also a tree operator.

The proof strategy is an adaptation of [19, Section 9]: indeed, the bound of Lemma 3.1 is an immediate consequence of the estimate (7) below. Having fixed a $k$-lacunary tree $T$, there holds

$$
\left\|T_{\mathrm{T}} f\right\|_{L^{q}(\mathbb{R} ; X)} \lesssim_{q} \lambda\left|I_{\mathrm{T}}\right|^{\frac{1}{q}}, \quad \lambda:=\sup _{I \in \mathcal{I}} \inf _{I} \mathrm{M}\left(|f|_{X}\right), \quad \mathcal{I}:=\left\{I_{P}: P \in \mathrm{T}\right\}, \quad 1<q<\infty .
$$

The estimate is uniform over tree operators $T_{\mathrm{T}}$.

By modulation invariance of (7), we may reduce to treating the case $\xi_{T}=0$. Then, estimate (7) will be obtained as a consequence of the next lemma.

7.4. Lemma. Let $\mathrm{T}$ be a k-lacunary tree with $\xi_{\mathrm{T}}=0, T_{\mathrm{T}}$ be a tree operator. For each J-dyadic interval $K \subset \mathbb{R}$ there exists a constant $a_{K}$ with the property that

$$
\left\|\mathbf{1}_{K}\left(T_{\mathrm{T}} f-a_{K}\right)\right\|_{L^{1, \infty}(\mathbb{R} ; X)} \lesssim \lambda|K|
$$

with bound independent of $K, T_{\top}$ and $\mathrm{T}$. In particular, if $\ell(K) \geq \ell\left(I_{\top}\right)$ we may take $a_{K}=0$. 
We use Lemma 7.1 to finish the proof of (7). Fix a tree operator $T_{\mathrm{T}}$. Then, referring to (7),

$$
\left\|T_{\mathrm{T}} f\right\|_{L^{q(\mathbb{R} ; X)}}=\left\|\gamma_{I_{\top}}^{-1} \tilde{T}_{\mathrm{T}} f\right\|_{L^{q}(\mathbb{R} ; X)} \lesssim \sum_{v \in \mathbb{Z}}\langle v\rangle^{-100}\left\|\mathbf{1}_{T_{\mathrm{T}}^{+v}} \tilde{T}_{\mathrm{T}} f\right\|_{L^{q}(\mathbb{R} ; X)}
$$

But, Lemma 7.1 applied to $\tilde{T}$ together with the John-Strömberg inequality yields the two estimates

$$
\left\|\mathbf{1}_{I_{\mathrm{T}}^{+v}} \tilde{T}_{\mathrm{T}} f\right\|_{L^{1, \infty}(\mathbb{R} ; X)} \lesssim \lambda\left|I_{\mathrm{T}}\right|, \quad\left\|\tilde{T}_{\mathrm{T}} f\right\|_{\mathrm{BMO}(\mathbb{R} ; X)} \lesssim \lambda,
$$

which together with the John-Nirenberg inequality tell us that

$$
\left\|\mathbf{1}_{I_{\mathrm{T}}^{+v}} \tilde{T}_{\mathrm{T}} f\right\|_{L^{q}(\mathbb{R} ; \mathcal{X})} \lesssim \lambda\left|I_{\mathrm{T}}\right|^{\frac{1}{q}} .
$$

A combination of (7) and (7) finally yields (7).

Proof of Lemma 7.1. We fix a tree operator and use the local notation

$$
T_{\mathrm{T}} f=\sum_{I \in I} S_{I} f
$$

where $S_{I}=S_{P_{k}} \in \mathbb{S}_{P_{k}}$ for the unique tri-tile $P \in \mathrm{T}$ with $I_{P}=I$.

We begin the proof with the definition of the constant $a_{K}$. This constant comes from the large scales contribution on $K$, that is the intervals

$$
\mathcal{I}^{\text {low }}=\{I \in \mathcal{I}: \ell(I)>\ell(K)\} .
$$

For $n \in \mathbb{N}$ let $K^{(n)}$ be the $n$-th $J$-dyadic parent of $K$. Then if $I \in \mathcal{I}_{\text {low }}$, it must be $I=K^{(n)+v}$ for some $n \in \mathbb{N}, v \in \mathbb{Z}$. We define

$$
a_{K}=\sum_{n \geq 1} \sum_{v \in \mathbb{Z}} S_{K^{(n)+v}} f(c(K))
$$

where we have simply set $S_{K^{(n)+v}}=0$ if $K^{(n)+v} \notin \mathcal{I}$. Clearly, the second claim now follows from the first, as $\mathcal{I}_{\text {low }}$ is empty, whence $a_{K}$ is zero, when $\ell(K) \geq \ell\left(I_{T}\right)$.

We continue with the proof of (7.1) We claim that

$$
\mathbf{1}_{K} \sum_{n \geq 1} \sum_{v \in \mathbb{Z}}\left|S_{K^{(n)+v}} f(c(K))-S_{K^{(n)+v}} f(x)\right|_{X} \lesssim \lambda .
$$

Indeed, denoting by $F=|f|_{\mathcal{X}}$, by $u_{n, v}$ the kernel of $T_{K^{(n)+v}}$ and by $\chi_{n, v}=\chi_{K^{(n)+v}}$ for simplicity, and using the kernel estimates for $u_{n, v}$ and the extra decay in $v$, we have for $x \in K$

$$
\begin{aligned}
& \left|S_{K^{(n)+v}} f(c(K))-S_{K^{(n)+v}} f(x)\right| \\
\leq & \left|\chi_{n, v}(x)-\chi_{n, v}(c(K))\right|\left(F *\left|u_{n, v}\right|(x)\right)+\int_{x}^{c(K)} F *\left|D u_{n, v}\right|(z) \mathrm{d} z \\
\lesssim & \langle v\rangle^{-100} 2^{-n} \inf _{K^{(n)+v}} \mathrm{M} F \leq\langle v\rangle^{-100} 2^{-n} \lambda,
\end{aligned}
$$

which is summable over $v, n$ in (7). The last estimate follows from the membership of $K^{(n)+v}$ to $\mathcal{I}$.

We now come to the small scales. We first deal with the contribution of the intervals

$$
I_{n, v}^{\text {high }}=\left\{I \in \mathcal{I}: \ell(I)=2^{-n} \ell(K), I \subset K^{+v}\right\}, \quad n \geq 0, v \in \mathbb{Z},|v|>1 .
$$


Notice that this excludes the intervals $\mathcal{I}^{\text {high }}=\{I \in \mathcal{I}: I \subset 3 K\}$ which will be handled as the main term. The $\mathcal{I}_{n, v}^{\text {high }}$ are tail terms: in fact, with the same notations as before, if $x \in K$ and $I \in I_{n, v}^{\text {high }}$

$$
\left|\chi_{I}(x)\right|\left(F *\left|u_{n, v}\right|(x)\right) \lesssim\left(v 2^{n}\right)^{-100} \sum_{t \geq 0} 2^{-100 t}\langle F\rangle_{\left[x-2^{t+1} \ell(I), x+2^{t+1} \ell(I)\right]}
$$

As, for $x \in K$,

$$
\langle F\rangle_{\left[x-2^{t+1} \ell(I), x+2^{t+1} \ell(I)\right]} \lesssim \begin{cases}\frac{v \ell(K)}{2^{t} \ell(I)}\langle F\rangle_{\left[x-2^{10} v \ell(K), x+2^{10} v \ell(K)\right]} \leq\left(v 2^{n}\right) \inf _{x \in I} \mathrm{M} F(x) & 2^{t+1} \ell(I) \leq v \ell(K) \\ \inf _{x \in I} \mathrm{M} F(x) & 2^{t+1} \ell(I)>v \ell(K)\end{cases}
$$

we obtain by summation of (7) that

$$
\mathbf{1}_{K} \sum_{n \geq 0} \sum_{|v| \geq 2} \sum_{I \in I_{n, v}^{\text {high }}}\left|S_{I} f\right|_{\mathcal{X}} \lesssim \lambda .
$$

We are left to estimate the contribution of $\mathcal{I}^{\text {high }}$. The union of the intervals $I^{\text {hi }}$ is contained in $3 K$. By possibly splitting $I^{\text {high }}$ into three collections and replacing $I \in I^{\text {high }}$ with the corresponding smoothing interval from one of three shifted dyadic grids, so that the union is still contained in $18 K$, we can achieve the property that if $I, L \in I^{\text {high }}$ and $I \subset L$ then $3 I \subset L$.

Let now $L \in \mathcal{L}$ be the collection of those $L \in \mathcal{I}^{\text {high }}$ which are maximal with respect to inclusion and $\mathcal{I}(L)=\left\{I \in \mathcal{I}^{\text {high }}: I \subsetneq L\right\}$. First we remove the tops. It is immediate to bound

$$
\sum_{L \in \mathcal{L}}\left\|S_{L} f\right\|_{L^{1}(\mathbb{R} ; \mathcal{X})} \lesssim \sum_{L \in \mathcal{L}}|L| \inf _{L} \mathrm{M} F \lesssim \lambda|K| .
$$

We estimate one more tail term. For $n \geq 1$ let $\mathcal{I}^{n}(L)=\left\{I \in \mathcal{I}(L): \ell(I)=2^{-n} \ell(L)\right\}$. For each $I \in I^{n}(L)$, let $z_{I}$ be the least nonnegative integer $z$ such that $(I \pm z \ell(I)) \cap(\mathbb{R} \backslash L) \neq \varnothing$. As $3 I \subset L$, we have $z_{I} \geq 1$. Furthermore for each integer $z \geq 1$, there are at most two intervals $I \in \mathcal{I}^{n}(L)$ with $z_{I}=z$. As for $x \in \mathbb{R} \backslash L$ we have $\operatorname{dist}(x, I) \geq \ell(I)$, there holds

$$
\begin{aligned}
\mathbf{1}_{\mathbb{R} \backslash L}(x)\left|S_{I} f(x)\right|_{X} & \lesssim\left\langle\frac{\operatorname{dist}(x, I)}{\ell(I)}\right\rangle^{-100} \sup _{s \gtrsim \ell(I)} \frac{1}{\left|B_{S}(x)\right|} \int_{B_{S}(x)} F \lesssim\left\langle\frac{\operatorname{dist}(x, I)}{\ell(I)}\right\rangle^{-99} \inf _{I} \mathrm{M} F \\
& \lesssim\left\langle\frac{\operatorname{dist}(x, I)}{\ell(I)}\right\rangle^{-90} z_{I}^{-9} \lambda
\end{aligned}
$$

Integrating over $\mathbb{R} \backslash L$ the last display for each $I$-summand, we have

$$
\sum_{n \geq 1} \sum_{I \in \mathcal{I}^{n}(L)}\left\|\mathbf{1}_{\mathbb{R} \backslash L} S_{I} f\right\|_{L^{1}(\mathbb{R} ; X)} \lesssim \lambda \sum_{n \geq 1} \sum_{I \in I^{n}(L)} z_{I}^{-9}|I| \lesssim \lambda \sum_{n \geq 1} \sum_{z \geq 1} z^{-9} 2^{-n}|L| \lesssim \lambda|L|
$$

whence

$$
\sum_{L \in \mathcal{L}} \sum_{I \in \mathcal{I}(L)}\left\|\mathbf{1}_{\mathbb{R} \backslash L} S_{I} f\right\|_{L^{1}(\mathbb{R} ; X)} \lesssim \lambda \sum_{L \in \mathcal{L}}|L| \lesssim \lambda|K|
$$


We are left to estimate the main term. Using disjointness of the supports of the summands below

$$
\left\|\sum_{L \in \mathcal{L}} \mathbf{1}_{L} \sum_{I \in \mathcal{I}(L)} S_{I} f\right\|_{L^{1, \infty}(\mathbb{R} ; \mathcal{X})} \leq \sum_{L \in \mathcal{L}}\left\|\sum_{I \in \mathcal{I}(L)} S_{I} f\right\|_{L^{1, \infty}(\mathbb{R} ; \mathcal{X})} .
$$

To estimate each summand on the right hand side of the last display, we use the localization trick. Referring to (7), set $\bar{S}_{I} g:=S_{I}\left(\gamma_{L} g\right)$. We then have

$$
\begin{aligned}
& \sum_{L \in \mathcal{L}}\left\|\sum_{I \in \mathcal{I}(L)} S_{I} f\right\|_{L^{1, \infty}(\mathbb{R} ; \mathcal{X})}=\sum_{L \in \mathcal{L}}\left\|\sum_{I \in \mathcal{I}(L)} \tilde{S}_{I}\left(\gamma_{L}^{-1} f\right)\right\|_{L^{1, \infty}(\mathbb{R} ; \mathcal{X})} \\
\lesssim & \sum_{L \in \mathcal{L}}\left\|\gamma_{L}^{-1} f\right\|_{L^{1}(\mathbb{R} ; \mathcal{X})} \lesssim \sum_{L \in \mathcal{L}}|L| \inf _{L} \mathrm{M} F \lesssim \lambda|K|
\end{aligned}
$$

as each $\bar{S}_{I} \in \mathbb{S}_{P}$ where $P \in \mathrm{T}$ is the unique tri-tile with $I_{P}=I$, and therefore each $L$-th summand on the right hand side of the first line is a Calderón-Zygmund operator. We achieve (7.1) by putting together (7), (7), (7), (7), (7), (7) and (7). The proof of the lemma is then complete.

\section{REFERENCES}

[1] Alex Amenta, Emiel Lorist, and Mark Veraar, Fourier multipliers in Banach function spaces with UMD concavifications, Trans. Amer. Math. Soc. 371 (2019), no. 7, 4837-4868. MR3934469 $\uparrow 4$

[2] Alex Amenta and Gennady Uraltsev, Banach-valued modulation invariant Carleson embeddings and outer- $L^{p}$ spaces: the Walsh case, preprint arXiv:1905.08681 (2019May), arXiv:1905.08681, available at 1905.08681. $\uparrow 4,6$

[3] Alex Amenta and Gennady Uraltsev, The bilinear Hilbert transform on UMD spaces, preprint arXiv:1909.06416 (2019May). $\uparrow 6$

[4] Cristina Benea and Camil Muscalu, Multiple vector-valued inequalities via the helicoidal method, Anal. PDE 9 (2016), no. 8, 1931-1988. MR3599522 $\uparrow 4$

[5] Jean Bourgain, Some remarks on Banach spaces in which martingale difference sequences are unconditional, Ark. Mat. 21 (1983), no. 2, 163-168. MR727340 44

[6] Donald L. Burkholder, A geometric condition that implies the existence of certain singular integrals of Banachspace-valued functions, Conference on harmonic analysis in honor of Antoni Zygmund, Vol. I, II (Chicago, Ill., 1981), 1983, pp. 270-286. MR730072 $\uparrow 4$

[7] Alejandro J. Castro and Tuomas P. Hytönen, Bounds for partial derivatives: necessity of UMD and sharp constants, Math. Z. 282 (2016), no. 3-4, 635-650. MR3473635 44

[8] David Cruz-Uribe and José María Martell, Limited range multilinear extrapolation with applications to the bilinear Hilbert transform, Math. Ann. 371 (2018), no. 1-2, 615-653. MR3788859 $\uparrow 4$

[9] Amalia Culiuc, Francesco Di Plinio, and Yumeng Ou, Domination of multilinear singular integrals by positive sparse forms, J. Lond. Math. Soc. (2) 98 (2018), no. 2, 369-392. MR3873113 $\uparrow 4,11$

[10] Ciprian Demeter and Christoph Thiele, On the two-dimensional bilinear Hilbert transform, Amer. J. Math. 132 (2010), no. 1, 201-256. MR2597511 $\uparrow 5,12$

[11] Francesco Di Plinio, Kangwei Li, Henri Martikainen, and Emil Vuorinen, Multilinear operator-valued Calderón-Zygmund theory, preprint, arXiv:1908.07233 (2019). $\uparrow 4$

[12] Francesco Di Plinio, Kangwei Li, Henri Martikainen, and Emil Vuorinen, Multilinear singular integrals on non-commutative $L^{p}$ spaces, preprint, arXiv:1905.02139 (2019). $\uparrow 4,5,6,10,12,14,15,16$

[13] Francesco Di Plinio and Yumeng Ou, Banach-valued multilinear singular integrals, Indiana Univ. Math. J. 67 (2018), no. 5, 1711-1763. MR3875242 $\uparrow 4$

[14] Stefan Geiss, Stephen Montgomery-Smith, and Eero Saksman, On singular integral and martingale transforms, Trans. Amer. Math. Soc. 362 (2010), no. 2, 553-575. MR2551497 $\uparrow 4$ 
[15] Timo S. Hänninen and Tuomas P. Hytönen, Operator-valued dyadic shifts and the T(1) theorem, Monatsh. Math. 180 (2016), no. 2, 213-253. MR3502626 个4

[16] Tuomas Hytönen and Pierre Portal, Vector-valued multiparameter singular integrals and pseudodifferential operators, Adv. Math. 217 (2008), no. 2, 519-536. MR2370274 (2009e:42027) $\uparrow 4$

[17] Tuomas Hytönen, Jan van Neerven, Mark Veraar, and Lutz Weis, Analysis in Banach spaces. Vol. I. Martingales and Littlewood-Paley theory, Ergebnisse der Mathematik und ihrer Grenzgebiete. 3. Folge. A Series of Modern Surveys in Mathematics [Results in Mathematics and Related Areas. 3rd Series. A Series of Modern Surveys in Mathematics], vol. 63, Springer, Cham, 2016. MR3617205 $\uparrow 2,4$

[18] Tuomas P. Hytönen, On operator-multipliers for mixed-norm $L^{\bar{p}}$ spaces, Arch. Math. (Basel) 85 (2005), no. 2, 151-155. MR2161804 (2006c:42011) $\uparrow 4$

[19] Tuomas P. Hytönen and Michael T. Lacey, Pointwise convergence of vector-valued Fourier series, Math. Ann. 357 (2013), no. 4, 1329-1361. MR3124934 个2, 4, 5, 6, 11, 12, 24, 26, 27, 28, 29

[20] Tuomas P. Hytönen and Michael T. Lacey, Pointwise convergence of Walsh-Fourier series of vector-valued functions, Math. Res. Lett. 25 (2018), no. 2, 561-580. MR3826835 $\uparrow 2,4$

[21] Tuomas P. Hytönen, Michael T. Lacey, and Ioannis Parissis, The vector valued quartile operator, Collect. Math. 64 (2013), no. 3, 427-454. MR3084406 ^4, 6, 10, 12

[22] Tuomas P. Hytönen, Michael T. Lacey, and Ioannis Parissis, A variation norm Carleson theorem for vectorvalued Walsh-Fourier series, Rev. Mat. Iberoam. 30 (2014), no. 3, 979-1014. MR3254998 个4, 12

[23] Marius Junge and Quanhua Xu, Representation of certain homogeneous Hilbertian operator spaces and applications, Invent. Math. 179 (2010), no. 1, 75-118. MR2563760 个4

[24] Nigel Kalton and Lutz Weis, The $H^{\infty}$-calculus and sums of closed operators, Math. Ann. 321 (2001), no. 2, 319-345. MR1866491 $\uparrow 4$

[25] Michael Lacey and Christoph Thiele, $L^{p}$ estimates on the bilinear Hilbert transform for $2<p<\infty$, Ann. of Math. (2) 146 (1997), no. 3, 693-724. MR1491450 ^2, 3, 11

[26] Michael Lacey and Christoph Thiele, On Calderón's conjecture, Ann. of Math. (2) 149 (1999), no. 2, 475496. MR1689336 $\uparrow 2,11$

[27] Camil Muscalu, Terence Tao, and Christoph Thiele, Multi-linear operators given by singular multipliers, J. Amer. Math. Soc. 15 (2002), no. 2, 469-496. MR1887641 $\uparrow 2,9,28$

[28] Camil Muscalu, Terence Tao, and Christoph Thiele, Uniform estimates on multi-linear operators with modulation symmetry, 2002, pp. 255-309. Dedicated to the memory of Tom Wolff. MR1979774 $\uparrow 5,8,9,10$, $11,12,13,14,16,17,18,19,20,21,22,23,26,28$

[29] Gilles Pisier and Quanhua Xu, Non-commutative $L^{p}$-spaces, Handbook of the geometry of Banach spaces, Vol. 2, 2003, pp. 1459-1517. MR1999201 个5

[30] Prabath Silva, Vector-valued inequalities for families of bilinear Hilbert transforms and applications to biparameter problems, J. Lond. Math. Soc. (2) 90 (2014), no. 3, 695-724. MR3291796 $\uparrow 4$

[31] Elias M. Stein, Harmonic analysis: real-variable methods, orthogonality, and oscillatory integrals, Princeton Mathematical Series, vol. 43, Princeton University Press, Princeton, NJ, 1993. With the assistance of Timothy S. Murphy, Monographs in Harmonic Analysis, III. MR1232192 (95c:42002) 111

[32] Christoph Thiele, Wave packet analysis, CBMS Regional Conference Series in Mathematics, vol. 105, Published for the Conference Board of the Mathematical Sciences, Washington, DC; by the American Mathematical Society, Providence, RI, 2006. MR2199086 $\uparrow 9,10$

[33] Lutz Weis, Operator-valued Fourier multiplier theorems and maximal L $L_{p}$-regularity, Math. Ann. 319 (2001), no. 4, 735-758. MR1825406 $\uparrow 4$ 